\title{
Elimination of the Incompleteness of Classical Dynamics
}

\author{
G. A. Skorobogatov", S. I. Svertilov \\ Dept of Chemistry, St.Petersburg State University, Universitetskii Prosp., 26, St.Petersburg 198504, Russia \\ *Corresponding Author: skorgera@rambler.ru
}

Copyright (C) 2013 Horizon Research Publishing All rights reserved.

\begin{abstract}
We have deductively and rigorously confirmed the Poincaré recurrence theorem also for bifurcating and branching solutions of differential equations. We have shown that the standard Newton mechanics (SNM) is incompatible with the Boltzmann H-theorem (BHT), thus being incomplete. Metamathematics demands that the primary axioms of SNM should be changed. It appears that BHT is compatible with the realistic mechanics, in which the standard Liouville equation (SLE) is replaced by the complete Liouville equation reducible to the SLE only for stable motions.
\end{abstract}

Keywords Classical Dynamics

\section{The Boltzmann H-theorem}

Let us consider a gas of several types of particles in a rigid container of volume V. We denote these types by indices $a$ or $b$. If we introduce $\mathrm{f}_{\mathrm{a}}(\overrightarrow{\mathrm{p}}, \overrightarrow{\mathrm{r}}, \mathrm{t})$, the distribution function of particles with momenta $\overrightarrow{\mathrm{p}}$ and coordinates $\overrightarrow{\mathrm{r}}$ at time $t$, then for the density of particles of type $a$ we obtain

$$
\int f_{a} d \vec{p}_{a}=n_{a}
$$

For the full number of particles of type $a$ one gets

$$
\int f_{a}\left(\vec{p}_{a}, \vec{r}_{a}, t\right) d \vec{r}_{a} d \vec{p}_{a}=N_{\mathrm{a}} .
$$

The inner degrees of freedom of gas molecules can be taken into account by introducing an additional argument in the distribution function: $f_{a}\left(\vec{p}_{a}, \vec{r}_{a}, s, t\right)$. Molecules are quantum objects, so their inner states are characterized by discrete series of quantum numbers. Thus, the molecules of type $a$ in different inner states can be considered as particles of different types, and it is more convenient to introduce a set of functions $f_{a, s}\left(\vec{p}_{a}, \vec{r}_{a}, t\right)$ instead of one distribution function $f_{a}\left(\vec{p}_{a}, \vec{r}_{a}, s, t\right)$.

In 1871, L. Boltzmann proposed the following integro-differential equation for the time evolution of the distribution function of a gas [1]:

$$
\begin{aligned}
& \frac{\partial f_{a}\left(\vec{p}_{a}, \vec{r}_{a}, t\right)}{\partial t}+\vec{v}_{a} \frac{\partial f_{a}\left(\vec{p}_{a}, \vec{r}_{a}, t\right)}{\partial \vec{r}_{a}}+\vec{F}_{a} \frac{\partial f_{a}\left(\vec{p}_{a}, \vec{r}_{a}, t\right)}{\partial \vec{p}_{a}}= \\
& =\sum_{b} \int \ldots \int\left\{f_{a}\left(\vec{p}_{a}^{\prime}, \vec{r}_{a}, t\right) f_{b}\left(\vec{p}_{b}^{\prime}, \vec{r}_{b}, t\right)-f_{a}\left(\vec{p}_{a}, \vec{r}_{a}, t\right) f_{b}\left(\vec{p}_{b}, \vec{r}_{b}, t\right)\right\} \times \\
& \times W_{a b}\left(\vec{p}_{a}, \vec{p}_{b} \mid \vec{p}_{a}^{\prime}, \vec{p}_{b}^{\prime}\right) \delta\left(\vec{p}_{a}+\vec{p}_{b}-\vec{p}_{a}^{\prime}-\vec{p}_{b}^{\prime}\right) \delta\left(E_{a}+E_{b}-E_{a}^{\prime}-E_{b}^{\prime}\right) d \vec{p}_{b} d \vec{p}_{a}^{\prime} d \vec{p}_{b}^{\prime},
\end{aligned}
$$

where $\overrightarrow{\mathrm{v}}_{\mathrm{a}}$ is the velocity of a particle of type $a, \overrightarrow{\mathrm{F}}_{\mathrm{a}}$ is the external force acting on a particle of type $a, \delta$ is the Dirac delta function. The product

$$
\mathrm{W}_{\mathrm{ab}}\left(\overrightarrow{\mathrm{p}}_{\mathrm{a}}, \overrightarrow{\mathrm{p}}_{\mathrm{b}} \mid \overrightarrow{\mathrm{p}}_{\mathrm{a}}^{\prime}, \overrightarrow{\mathrm{p}}_{\mathrm{b}}^{\prime}\right) \delta\left(\overrightarrow{\mathrm{p}}_{\mathrm{a}}+\overrightarrow{\mathrm{p}}_{\mathrm{b}}-\overrightarrow{\mathrm{p}}_{\mathrm{a}}^{\prime}-\overrightarrow{\mathrm{p}}_{\mathrm{b}}^{\prime}\right) \delta\left(\mathrm{E}_{\mathrm{a}}+\mathrm{E}_{\mathrm{b}}-\mathrm{E}_{\mathrm{a}}^{\prime}-\mathrm{E}_{\mathrm{b}}^{\prime}\right) \equiv \mathrm{w}_{\mathrm{ab}}\left(\overrightarrow{\mathrm{p}}_{\mathrm{a}}, \overrightarrow{\mathrm{p}}_{\mathrm{b}} \mid \overrightarrow{\mathrm{p}}_{\mathrm{a}}^{\prime}, \overrightarrow{\mathrm{p}}_{\mathrm{b}}^{\prime}\right)
$$

is the probability of a transition, as a result of collision, of two particles of types a and b from the initial state with energies and 
momenta $E_{a}, \overrightarrow{\mathrm{p}}_{\mathrm{a}}, E_{b}, \overrightarrow{\mathrm{p}}_{\mathrm{b}}$ to the final state with energies and momenta $\mathrm{E}_{\mathrm{a}}^{\prime}, \overrightarrow{\mathrm{p}}_{\mathrm{a}}^{\prime}, \mathrm{E}_{\mathrm{b}}^{\prime} \overrightarrow{\mathrm{p}}_{\mathrm{b}}^{\prime}$.

The external force can be, for example, the Earth gravitation force $\overrightarrow{\mathrm{F}}_{\mathrm{a}}=\mathrm{m}_{\mathrm{a}} \overrightarrow{\mathrm{g}}$, where $m_{\mathrm{a}}$ is the mass of a type $a$ molecule. Or, if the particles are charged, they will be affected by the Lorenz force in the presence of external electric and magnetic fields.

The quantity (2) can be written in the form:

$$
W_{a b}\left(\vec{p}_{a}, \vec{p}_{b} \mid \vec{p}_{a}^{\prime}, \vec{p}_{b}^{\prime}\right) d \vec{p}_{a}^{\prime}=v_{a b} d \sigma_{a b} d E_{a}^{\prime},
$$

where $\mathrm{d} \sigma_{\mathrm{ab}}=\mathrm{d} \sigma_{\mathrm{ab}}\left(\mathrm{v}_{\mathrm{ab}}, \theta, \varphi\right)$ is the effective cross-section of the scattering of particles of $a$ and $b$ types in the solid angle $\mathrm{do}_{\mathrm{n}}=\sin \theta \mathrm{d} \theta \mathrm{d} \varphi ; \mathrm{v}_{a b}$ is the relative velocity of colliding molecules: $\overrightarrow{\mathrm{v}}_{\mathrm{ab}}=\frac{\overrightarrow{\mathrm{p}}_{\mathrm{a}}}{\mathrm{m}_{\mathrm{a}}}-\frac{\overrightarrow{\mathrm{p}}_{\mathrm{b}}}{\mathrm{m}_{\mathrm{b}}}$. Then the kinetic equation (1) can be rewritten in a more compact form:

$$
\frac{\partial f_{a}}{\partial t}+\vec{v}_{a} \frac{\partial f_{a}}{\partial \vec{r}_{a}}+\vec{F}_{a} \frac{\partial f_{a}}{\partial \vec{p}_{a}}=\sum_{b} \int\left\{f_{a}^{\prime} f_{b}^{\prime}-f_{a} f_{b}\right\} v_{a b} d \sigma_{a b} d \vec{p}_{b} .
$$

Let us consider a gas in the absence of external forces and let us assume that the particle distribution be space-homogeneous. Then the equation (4) is simplified to the following form:

$$
\frac{\partial f_{a}}{\partial t}=\sum_{b} \int\left\{f_{a}^{\prime} f_{b}^{\prime}-f_{a} f_{b}\right\} v_{a b} d \sigma_{a b} d \vec{p}_{b}
$$

Let us introduce the so-called Boltzmann H-function:

$$
H(t)=\sum_{a} \int f_{a}\left(\vec{p}_{a}, t\right) \cdot \ln \left[f_{a}\left(\vec{p}_{a}, t\right)\right] d \vec{p}_{a}
$$

Differentiation of the both parts of (6) gives

$$
\frac{d H}{d t}=\sum_{a} \int\left[1+\ln f_{a}\right] \frac{\partial f_{a}}{\partial t} d \vec{p}_{a}
$$

The right part of (7) can be modified with the help of (5):

$$
\begin{aligned}
& \sum_{a b} \int\left[1+\ln f_{a}\right]\left\{f_{a}^{\prime} f_{b}^{\prime}-f_{a} f_{b}\right\} v_{a b} d \sigma_{a b} d \vec{p}_{a} d \vec{p}_{b}= \\
& =\frac{1}{2} \sum_{a b} \int\left[2+\ln \left(f_{a} f_{b}\right)\right]\left\{f_{a}^{\prime} f_{b}^{\prime}-f_{a} f_{b}\right\} v_{a b} d \sigma_{a b} d \vec{p}_{a} d \vec{p}_{b}= \\
& =\frac{1}{4} \sum_{a b} \int\left[2+\ln \left(f_{a} f_{b}\right)-2-\ln \left(f_{a}^{\prime} f_{b}^{\prime}\right)\right]\left\{f_{a}^{\prime} f_{b}^{\prime}-f_{a} f_{b}\right\} v_{a b} d \sigma_{a b} d \vec{p}_{a} d \vec{p}_{b},
\end{aligned}
$$

where in the last line we use the equality $d \vec{p}_{a} d \vec{p}_{b}=d \vec{p}_{a}^{\prime} d \vec{p}_{b}^{\prime}$ and the fact that in (3) the relative velocity $v_{a b}$ and differential cross-section $\sigma_{\mathrm{ab}}$ do not change under replacement of momenta before collision (undashed) by those after collision (dashed). Thus, (7) can be rewritten in the form:

$$
\frac{d H}{d t}=-\frac{1}{4} \sum_{a b} \int\left(\ln \frac{f_{a}^{\prime} f_{b}^{\prime}}{f_{a} f_{b}}\right)\left[f_{a}^{\prime} f_{b}^{\prime}-f_{a} f_{b}\right] v_{a b} d \sigma_{a b} d \vec{p}_{a} d \vec{p}_{b}
$$

The integrand in (9) is of type $F(x, y)=(x-y) \cdot \ln \frac{x}{y}$, so is always non-negative, Consequently, dH/dt is always negative:

$$
\frac{\mathrm{dH}}{\mathrm{dt}} \leq 0 .
$$


L. Boltzmann was the first to pay attention to the fact that, if one associates the function $-\mathrm{H}$ with the entropy $\mathrm{S}$, then from the inequality (10) it follows the law of entropy growth:

$$
\frac{\mathrm{dS}}{\mathrm{dt}} \geq 0
$$

\section{N.N. Bogolubov's Method}

Let us consider a system of $N$ identical particles of mass $m$. The boundary effects will be neglected as we pass to the limit of an infinitely large volume and infinite number of particles, under the condition of a finite average particle number density. That means that we take the Hamilton function as follows:

$$
H=\frac{1}{2 m} \sum_{i=1}^{N} p_{i}^{2}+\frac{1}{2} \sum_{\substack{i, j=1 \\(i \neq j)}}^{N} \Phi\left(\left|\vec{r}_{i}-\vec{r}_{j}\right|\right)
$$

where $\Phi$ is a central pair interaction potential.

Then we introduce the most detailed $N$-particle distribution function

$$
\mathrm{F}_{\mathrm{N}}\left(\mathrm{t}, \overrightarrow{\mathrm{r}}_{1}, \ldots, \overrightarrow{\mathrm{r}}_{\mathrm{N}}, \overrightarrow{\mathrm{p}}_{1}, \ldots, \overrightarrow{\mathrm{p}}_{\mathrm{N}}\right)
$$

of coordinates $\overrightarrow{\mathrm{r}}_{\mathrm{i}}$ and momenta $\overrightarrow{\mathrm{p}}_{\mathrm{i}}$ of all particles. The function (13) is normalized to unity:

$$
\int \mathrm{F}_{\mathrm{N}}\left(\mathrm{t}, \overrightarrow{\mathrm{r}}_{1}, \ldots, \overrightarrow{\mathrm{p}}_{\mathrm{N}}\right) \mathrm{d} \overrightarrow{\mathrm{r}}_{1}, \ldots, \mathrm{d} \overrightarrow{\mathrm{p}}_{\mathrm{N}}=1
$$

According to the Liouville theorem (p. 347 in [2]), the function (13) obeys the equation

$$
\frac{d F_{N}}{d t} \equiv \frac{\partial F_{N}}{\partial t}+\sum_{i=1}^{N}\left\{\frac{\partial F_{N}}{\partial \vec{r}_{i}} \dot{\vec{r}}_{i}+\frac{\partial F_{N}}{\partial \vec{p}_{i}} \dot{\vec{p}}_{i}\right\}=0
$$

Since $\dot{\overrightarrow{\mathrm{r}}}_{\mathrm{i}}=\frac{\partial \mathrm{H}}{\partial \overrightarrow{\mathrm{p}}_{\mathrm{i}}}$ and $\dot{\overrightarrow{\mathrm{p}}}_{\mathrm{i}}=-\frac{\partial \mathrm{H}}{\partial \overrightarrow{\mathrm{r}}_{\mathrm{i}}}$, the equation of motion (14) for the whole system of $N$ particles can be rewritten in the form of the Liouville equation:

$$
\frac{\partial F_{N}}{\partial t}=\left[H, F_{N}\right]
$$

where the symbol of the Poisson brackets is used:

$$
\left[H, F_{N}\right]=\sum_{i=1}^{N}\left\{\frac{\partial H}{\partial \vec{r}_{i}} \cdot \frac{\partial F_{N}}{\partial \vec{p}_{i}}-\frac{\partial H}{\partial \vec{p}_{i}} \cdot \frac{\partial F_{N}}{\partial \vec{r}_{i}}\right\} .
$$

The equation (15) is considered to describe uniquely the whole evolution of the system [2-4]. However, in the case of real gas consisting of billions and trillions of particles it is absolutely impossible to solve this equation both analytically and numerically. Therefore, one should sacrifice exactness and use a less detailed description of the system.

For that, let us introduce, on the basis of $\mathrm{F}_{\mathrm{N}}\left(\mathrm{t}, \overrightarrow{\mathrm{r}}_{1}, \ldots, \overrightarrow{\mathrm{r}}_{\mathrm{N}}, \overrightarrow{\mathrm{p}}_{1}, \ldots, \overrightarrow{\mathrm{p}}_{\mathrm{N}}\right)$, less detailed one-particle $\mathrm{F}_{1}\left(\mathrm{t}, \overrightarrow{\mathrm{r}}_{\mathrm{i}}, \overrightarrow{\mathrm{p}}_{\mathrm{i}}\right)$, two-particle $\mathrm{F}_{2}\left(\mathrm{t}, \overrightarrow{\mathrm{r}}_{\mathrm{i}}, \overrightarrow{\mathrm{r}}_{\mathrm{j}}, \overrightarrow{\mathrm{p}}_{\mathrm{i}}, \overrightarrow{\mathrm{p}}_{\mathrm{j}}\right)$, etc. distribution functions:

$$
\begin{aligned}
& \mathrm{F}_{1}\left(\mathrm{t}, \overrightarrow{\mathrm{r}}_{\mathrm{i}}, \overrightarrow{\mathrm{p}}_{\mathrm{i}}\right)=\mathrm{V} \int \mathrm{F}_{\mathrm{N}}\left(\mathrm{t}, \overrightarrow{\mathrm{r}}_{1}, \ldots, \overrightarrow{\mathrm{p}}_{\mathrm{N}}\right) \mathrm{d} \overrightarrow{\mathrm{r}}_{1} \ldots \mathrm{d} \overrightarrow{\mathrm{r}}_{\mathrm{i}-1} \mathrm{~d} \overrightarrow{\mathrm{r}}_{\mathrm{i}+1} \ldots \mathrm{d} \overrightarrow{\mathrm{r}}_{\mathrm{N}} \mathrm{d} \overrightarrow{\mathrm{p}}_{1} \ldots \mathrm{d} \overrightarrow{\mathrm{p}}_{\mathrm{i}-1} \mathrm{~d} \overrightarrow{\mathrm{p}}_{\mathrm{i}+1} \ldots \mathrm{d} \overrightarrow{\mathrm{p}}_{\mathrm{N}}, \\
& \mathrm{F}_{2}\left(\mathrm{t}, \overrightarrow{\mathrm{r}}_{\mathrm{i}}, \overrightarrow{\mathrm{r}}_{\mathrm{j}}, \overrightarrow{\mathrm{p}}_{\mathrm{i}}, \overrightarrow{\mathrm{p}}_{\mathrm{j}}\right)= \\
& =\mathrm{V}^{2} \int \mathrm{F}_{\mathrm{N}}\left(\mathrm{t}, \overrightarrow{\mathrm{r}}_{1}, \ldots, \overrightarrow{\mathrm{p}}_{\mathrm{N}}\right) \mathrm{d} \overrightarrow{\mathrm{r}}_{1} \ldots \mathrm{d} \overrightarrow{\mathrm{r}}_{\mathrm{i}-1} \mathrm{~d} \overrightarrow{\mathrm{r}}_{\mathrm{i}+1} \ldots \mathrm{d} \overrightarrow{\mathrm{r}}_{\mathrm{j}-1} \mathrm{~d} \overrightarrow{\mathrm{r}}_{\mathrm{j}+1} \ldots \mathrm{d} \overrightarrow{\mathrm{r}}_{\mathrm{N}} \mathrm{d} \overrightarrow{\mathrm{p}}_{1} \ldots \mathrm{d} \overrightarrow{\mathrm{p}}_{\mathrm{i}-1} \mathrm{~d} \overrightarrow{\mathrm{p}}_{\mathrm{i}+1} \ldots \mathrm{d} \overrightarrow{\mathrm{p}}_{\mathrm{j}-1} \mathrm{~d} \overrightarrow{\mathrm{p}}_{\mathrm{j}+1} \ldots \mathrm{d} \overrightarrow{\mathrm{p}}_{\mathrm{N}} \\
& F_{s}\left(t, \vec{r}_{1}, \ldots, \vec{r}_{s}, \vec{p}_{1}, \ldots, \vec{p}_{s}\right)=V^{s} \int F_{N}\left(t, \vec{r}_{1}, \ldots, \vec{r}_{N}, \vec{p}_{1}, \ldots, \vec{p}_{N}\right) d \vec{r}_{s+1} \ldots d \vec{r}_{N} d \vec{p}_{s+1} \ldots d \vec{p}_{N} .
\end{aligned}
$$


Let us integrate the equation (15) over phase spaces of $(N-S)$ particles:

$$
\frac{1}{\mathrm{~V}^{\mathrm{s}}} \cdot \frac{\partial \mathrm{F}_{\mathrm{s}}\left(\mathrm{t}, \overrightarrow{\mathrm{r}}_{1}, \ldots, \overrightarrow{\mathrm{p}}_{\mathrm{s}}\right)}{\partial \mathrm{t}}=\int\left[\mathrm{H}, \mathrm{F}_{\mathrm{N}}\right] \mathrm{d} \overrightarrow{\mathrm{r}}_{\mathrm{s}+1} \ldots \mathrm{d} \overrightarrow{\mathrm{p}}_{\mathrm{N}}
$$

Substituting here the function (12), we obtain [3, 4]:

$$
\begin{aligned}
& \frac{1}{V^{s}} \cdot \frac{\partial F_{s}}{\partial t}=-\frac{1}{V^{s}} \sum_{i=1}^{s} \frac{\vec{p}_{i}}{m} \cdot \frac{\partial F_{s}}{\partial \vec{r}_{i}}+\frac{1}{2 V^{s}} \sum_{\substack{i, j=1 \\
(i \neq j)}}^{s} \frac{\partial \Phi\left(\left|\vec{r}_{i}-\vec{r}_{j}\right|\right)}{\partial \vec{r}_{i}} \cdot \frac{\partial F_{s}}{\partial \vec{p}_{i}}+ \\
& +\frac{1}{V^{s+1}} \sum_{i=1}^{s} \int \sum_{j=s+1}^{N} \frac{\partial \Phi\left(\left|\vec{r}_{i}-\vec{r}_{j}\right|\right)}{\partial \vec{r}_{i}} \cdot \frac{\partial F_{s+1}\left(t, \vec{r}_{1}, \ldots, \vec{r}_{s}, \vec{r}_{j}, \vec{p}_{1}, \ldots, \vec{p}_{s}, \vec{p}_{j}\right)}{\partial \vec{p}_{i}} d \vec{r}_{j} d \vec{p}_{j}- \\
& -\frac{1}{V^{s+1}} \sum_{j=s+1}^{N} \int \frac{\vec{p}_{j}}{m} \cdot \frac{\partial F_{s+1}\left(t, \vec{r}_{1}, \ldots, \vec{r}_{s}, \vec{r}_{j}, \vec{p}_{1}, \ldots, \vec{p}_{s}, \vec{p}_{j}\right)}{\partial \vec{r}_{j}} d \vec{r}_{j} d \vec{p}_{j}+ \\
& +\frac{1}{V^{s+1}} \sum_{i=1}^{s} \sum_{j=s+1}^{N} \int \frac{\partial \Phi\left(\left|\vec{r}_{i}-\vec{r}_{j}\right|\right)}{\partial \vec{r}_{j}} \cdot \frac{\partial F_{s+1}\left(t, \vec{r}_{1}, \ldots, \vec{r}_{s}, \vec{r}_{j}, \vec{p}_{1}, \ldots, \vec{p}_{s}, \vec{p}_{j}\right)}{\partial \vec{p}_{j}} d \vec{r}_{j} d \vec{p}_{j}+ \\
& +\frac{1}{2 V^{s+2}} \sum_{i, j=s+1}^{N} \int \frac{\partial \Phi\left(\left|\vec{r}_{i}-\vec{r}_{j}\right|\right)}{\partial \vec{r}_{i}} \cdot \frac{\partial F_{s+2}\left(t, \vec{r}_{1}, \ldots, \vec{r}_{s}, \vec{r}_{i}, \vec{r}_{j}, \vec{p}_{1}, \ldots, \vec{p}_{s}, \vec{p}_{i}, \vec{p}_{j}\right)}{\partial \vec{p}_{i}} d \vec{r}_{i} d \vec{r}_{j} d \vec{p}_{i} d \vec{p}_{j} .
\end{aligned}
$$

The Hamilton function (12) does not take into account the walls of the container with gas. Thus, the presence of walls justifies the following boundary conditions:

$$
\left.\begin{array}{l}
\frac{\partial \mathrm{F}_{\mathrm{n}}}{\partial \overrightarrow{\mathrm{r}}_{\mathrm{j}}} \rightarrow 0 \text { when } \quad\left|\overrightarrow{\mathrm{r}}_{\mathrm{j}}\right| \rightarrow \infty \\
\frac{\partial \mathrm{F}_{\mathrm{n}}}{\partial \overrightarrow{\mathrm{p}}_{\mathrm{j}}} \rightarrow 0 \text { when } \quad\left|\overrightarrow{\mathrm{p}}_{\mathrm{j}}\right| \rightarrow \infty
\end{array}\right\}
$$

The boundary conditions (20) turn the three last terms in the equation (19) into zero. Besides, for subsystems of $s$ particles (s $<<\mathrm{N}$ ) we have

$$
\sum_{j=s+1}^{N} \int \frac{\partial \Phi_{i j}}{\partial \vec{r}_{i}} \cdot \frac{\partial F_{s+1}\left(t, \vec{r}_{1}, \ldots, \vec{r}_{s}, \vec{r}_{j}, \vec{p}_{1}, \ldots, \vec{p}_{s}, \vec{p}_{j}\right)}{\partial \vec{p}_{i}} d \vec{r}_{j} d \vec{p}_{j} \approx N \int \frac{\partial \Phi_{i, s+1}}{\partial \vec{r}_{i}} \cdot \frac{\partial F_{s+1}}{\partial \vec{p}_{i}} d \vec{r}_{s+1} d \vec{p}_{s+1}
$$

As a result, the equation (19) becomes much simpler:

$$
\frac{\partial \mathrm{F}_{\mathrm{s}}}{\partial \mathrm{t}}=\left[\mathrm{H}^{(\mathrm{s})}, \mathrm{F}_{\mathrm{s}}\right]+\frac{1}{\langle\mathrm{~V}\rangle} \sum_{\mathrm{i}=1}^{\mathrm{s}} \int \frac{\partial \Phi\left(\left|\overrightarrow{\mathrm{r}}_{\mathrm{i}}-\overrightarrow{\mathrm{r}}_{\mathrm{s}+1}\right|\right)}{\partial \overrightarrow{\mathrm{r}}_{\mathrm{i}}} \cdot \frac{\partial \mathrm{F}_{\mathrm{s}+1}}{\partial \overrightarrow{\mathrm{p}}_{\mathrm{i}}} \mathrm{d} \overrightarrow{\mathrm{r}}_{\mathrm{s}+1} \mathrm{~d} \overrightarrow{\mathrm{p}}_{\mathrm{s}+1}
$$

where $\langle\mathrm{V}\rangle$ is the average volume per one particle, and $\mathrm{H}^{(\mathrm{s})}$ is the Hamilton function for a closed system of $s$ particles.

Thus, from one equation (15) for the $6 N$-dimensional distribution function $F_{N}$, we obtain the chain of equations (21) for distribution functions $F_{1}, F_{2}, F_{3}, \ldots F_{N}$, where $1<<N^{\prime}<<N$. At that, the integrand in the equation (21) for $F_{s}$ contains the function $F_{s+l}$. That means that to obtain an exact solution one should solve simultaneously all the equations (21) for $\mathrm{s}=1$, $2, \ldots N^{\prime}$. However, even in $1 \mathrm{~mm}^{3}$ of gas under normal conditions there are $2,6 \cdot 10^{16}$ molecules, so the number $N^{\prime}$ is of the order of quadrillions, and the chain of equations (21) remains as unresolvable as the equation (15).

Therefore, one should once more sacrifice exactness and, on the basis of an appropriate model, break the chain (21) at some value $s$, choosing an analytical form for the first of neglected functions $\left(\mathrm{F}_{\mathrm{s}+1}\right)$. Besides N.N. Bogolubov [4], different ways of chain breaking were proposed by M. Born, G. Green, J. Kirkwood, J. Yvon, and other theoreticians [3]. The method of decoupling of correlation functions $\mathrm{F}_{1}, \ldots \mathrm{F}_{\mathrm{s}}$ in (14) is known as the BBGKY method.

The Boltzmann kinetic equations (1), (4) have the following general form: 


$$
\frac{\partial \mathrm{F}_{1}}{\partial \mathrm{t}}=\mathrm{L}\left(\mathrm{F}_{1}\right)
$$

where $L$ is a corresponding operator. In other words, the Boltzmann equation (1) is the chain (21) broken already at the first equation. To achieve any progress in comparison with the Boltzmann kinetic theory, one should retain at least two equations in the chain (21):

$$
\begin{gathered}
\frac{\partial \mathrm{F}_{1}\left(\mathrm{t}, \overrightarrow{\mathrm{r}}_{1}, \overrightarrow{\mathrm{p}}_{1}\right)}{\partial \mathrm{t}}=\left[\mathrm{H}^{(1)}, \mathrm{F}_{1}\left(\mathrm{t}, \overrightarrow{\mathrm{r}}_{1}, \overrightarrow{\mathrm{p}}_{1}\right)\right]+\frac{1}{\langle\mathrm{~V}\rangle} \int \frac{\partial \Phi\left(\left|\overrightarrow{\mathrm{r}}_{1}-\overrightarrow{\mathrm{r}}_{2}\right|\right)}{\partial \overrightarrow{\mathrm{r}}_{1}} \cdot \frac{\partial \mathrm{F}_{2}\left(\mathrm{t}, \overrightarrow{\mathrm{r}}_{1}, \overrightarrow{\mathrm{r}}_{2}, \overrightarrow{\mathrm{p}}_{1}, \overrightarrow{\mathrm{p}}_{2}\right)}{\partial \overrightarrow{\mathrm{p}}_{1}} \mathrm{~d}_{2} \mathrm{r}_{2} \overrightarrow{\mathrm{p}}_{2} ; \\
\frac{\partial F_{2}\left(t, \vec{r}_{1}, \vec{r}_{2}, \vec{p}_{1}, \vec{p}_{2}\right)}{\partial t}=\left[H^{(2)}, F_{2}\left(t, \vec{r}_{1}, \vec{r}_{2}, \vec{p}_{1}, \vec{p}_{2}\right)\right]+ \\
+\frac{1}{\langle V\rangle} \int\left\{\frac{\partial \Phi\left(\left|\vec{r}_{1}-\vec{r}_{3}\right|\right)}{\partial \vec{r}_{1}} \cdot \frac{\partial F_{3}\left(t, \vec{r}_{1}, \vec{r}_{2}, \vec{r}_{3}, \vec{p}_{1}, \vec{p}_{2}, \vec{p}_{3}\right)}{\partial \vec{p}_{1}}+\frac{\partial \Phi\left(\left|\vec{r}_{2}-\vec{r}_{3}\right|\right)}{\partial \vec{r}_{2}} \cdot \frac{\partial F_{3}\left(t, \vec{r}_{1}, \vec{r}_{2}, \vec{r}_{3}, \vec{p}_{1}, \vec{p}_{2}, \vec{p}_{3}\right)}{\partial \vec{p}_{2}}\right\} d \vec{r}_{3} d \vec{p}_{3},
\end{gathered}
$$

where an appropriate function should be substituted instead of $F_{3}$. N.N. Bogolubov [4] proposed the following approximation:

$$
\mathrm{F}_{3} \approx \frac{1}{3}\left\{\mathrm{~F}_{1}\left(\overrightarrow{\mathrm{r}}_{1}, \overrightarrow{\mathrm{p}}_{1}\right) \mathrm{F}_{2}\left(\overrightarrow{\mathrm{r}}_{2}, \overrightarrow{\mathrm{r}}_{3}, \overrightarrow{\mathrm{p}}_{2}, \overrightarrow{\mathrm{p}}_{3}\right)+\mathrm{F}_{1}\left(\overrightarrow{\mathrm{r}}_{2}, \overrightarrow{\mathrm{p}}_{2}\right) \mathrm{F}_{2}\left(\overrightarrow{\mathrm{r}}_{1}, \overrightarrow{\mathrm{r}}_{3}, \overrightarrow{\mathrm{p}}_{1}, \overrightarrow{\mathrm{p}}_{3}\right)+\mathrm{F}_{1}\left(\overrightarrow{\mathrm{r}}_{3}, \overrightarrow{\mathrm{p}}_{3}\right) \mathrm{F}_{2}\left(\overrightarrow{\mathrm{r}}_{1}, \overrightarrow{\mathrm{r}}_{2}, \overrightarrow{\mathrm{p}}_{1}, \overrightarrow{\mathrm{p}}_{2}\right)\right\}
$$

To obtain the kinetic equation (22), some approximation of the two-particle distribution function $\mathrm{F}_{2}$ is necessary. It can be seen from (23) that there is a certain correlation between changes in time of functions $F_{1}$ and $F_{2}$. If the time of matching of these changes $\left(\tau_{2}\right)$ is less than the characteristic time of the evolution of $\mathrm{F}_{1}$, one can introduce a model representation, in which the disturbed function $\mathrm{F}_{2}$ relaxes within the time interval $\tau_{2}$ in accordance with the value of the function $\mathrm{F}_{1}$, which remains nearly constant within this time interval. So, one can retain in $F_{2}$ only an implicit dependence on time via the more slowly changing function $\mathrm{F}_{1}$ :

$$
F_{2}=F_{2}\left(\vec{r}_{1}, \vec{r}_{2}, \vec{p}_{1}, \vec{p}_{2} ; F_{1}\right) .
$$

At that, in other functions $F_{s}(s=3,4, \ldots)$ of the chain (21), it is also sufficient to study only the "slow" time evolution $F_{s}=F_{s}$ $\left(\mathrm{F}_{1}\right)$.

To advance from the equation (23) to the Boltzmann equation (1), one should adopt the "factorization condition" in the infinite past $(\mathrm{t} \rightarrow-\infty)$ for all distribution functions $\mathrm{F}_{\mathrm{s}}(\mathrm{s}=2,3, \ldots)$ :

$$
\lim _{\mathrm{t} \rightarrow-\infty}\left\{\mathrm{F}_{\mathrm{s}}\left(\mathrm{t}, \mathrm{x}_{1}, \ldots, \mathrm{x}_{\mathrm{s}}\right)-\prod_{\mathrm{j}=1}^{\mathrm{s}} \mathrm{F}_{1}\left(\mathrm{t}, \mathrm{x}_{\mathrm{j}}\right)\right\}=0 .
$$

It is confessed in [3] (p. 27) that this condition is not connected with the dynamic approach and has the probabilistic nature of mass phenomena laws. This condition alone is sufficient to guarantee the thermodynamic irreversibility of a gas and the possibility to prove the Boltzmann $\mathrm{H}$-theorem. The function (25) under the initial condition (26) can be presented as the product

$$
\mathrm{F}_{2}=\mathrm{F}_{1}\left(\mathrm{t}, \overrightarrow{\mathrm{R}}_{1}^{(2)}, \overrightarrow{\mathrm{P}}_{1}^{(2)}\right) \cdot \mathrm{F}_{1}\left(\mathrm{t}, \overrightarrow{\mathrm{R}}_{2}^{(2)}, \overrightarrow{\mathrm{P}}_{2}^{(2)}\right)
$$

where

$$
\overrightarrow{\mathrm{R}}_{\mathrm{i}}^{(2)}=\lim _{\tau \rightarrow+\infty}\left\{\mathrm{S}_{-\tau}^{(2)} \overrightarrow{\mathrm{r}}_{\mathrm{i}}+\frac{\mathrm{S}_{-\tau}^{(2)} \overrightarrow{\mathrm{p}}_{\mathrm{i}}}{\mathrm{m}} \tau\right\} ; \quad \overrightarrow{\mathrm{P}}_{\mathrm{i}}^{(2)}=\lim _{\tau \rightarrow+\infty} \mathrm{S}_{-\tau}^{(2)} \overrightarrow{\mathrm{p}}_{\mathrm{i}}
$$

$\mathrm{S}_{\mathrm{t}}^{(\mathrm{s})}$ is the time evolution operator. It transforms the particles coordinates $\mathrm{x}_{1}, \ldots, \mathrm{X}_{\mathrm{s}}$ into those $\mathrm{X}_{1}, \ldots, \mathrm{X}_{\mathrm{s}}$ (which the particles will have in the time $t$ ), granted the system remains closed within this time interval. As a result, for a mechanical system described by the Hamiltonian (12) the equation (23) has the form

$$
\frac{\partial \mathrm{F}_{1}(\mathrm{t}, \overrightarrow{\mathrm{r}}, \overrightarrow{\mathrm{p}})}{\partial \mathrm{t}}=\left[\frac{\mathrm{p}^{2}}{2 \mathrm{~m}}, \mathrm{~F}_{1}(\mathrm{t}, \overrightarrow{\mathrm{r}}, \overrightarrow{\mathrm{p}})\right]+\frac{1}{\langle\mathrm{~V}\rangle} \int\left[\Phi\left(\left|\overrightarrow{\mathrm{r}}-\overrightarrow{\mathrm{r}}^{\prime}\right|\right), \mathrm{F}_{1}\left(\mathrm{t}, \overrightarrow{\mathrm{R}}^{(2)}, \overrightarrow{\mathrm{P}}^{(2)}\right) \mathrm{F}_{1}\left(\mathrm{t}, \overrightarrow{\mathrm{R}}^{(2)^{\prime}}, \overrightarrow{\mathrm{P}}^{(2)^{\prime}}\right)\right] \mathrm{d} \overrightarrow{\mathrm{r}}^{\prime} \mathrm{d} \overrightarrow{\mathrm{p}}^{\prime}
$$


Calculation of the Poisson brackets in (29) just gives the equation (1) without the term containing the external force $\overrightarrow{\mathrm{F}}_{\mathrm{a}}$, which has not been taken into account by us.

\section{The Poincare's Theorem. The Correlation Sphere According to G. A. Martynov}

An impression can be formed that the method of N. N. Bogolubov is logically irreproachable and completes the justification of the Boltzmann kinetic theory. However, it is not so. Moreover, the introduction of the hierarchy of BBGKY equations does not make it possible to answer principal questions posed to L. Boltzmann long ago by his opponents.

One of the hard problems of the kinetic theory that L. Boltzmann could not resolve logically rigorously is the Poincaré recurrence theorem. The theorem deals with the class of closed Hamiltonian systems. A unicomponent gas placed in a container with absolutely hard walls is an example of such systems, since for such gas there exists the Hamilton function (12), the total energy of gas particles is conserved (conservatism) and none of the particles go off to infinity (finiteness). Consider an area $\mathrm{A}$ of a positive measure in phase space and choose a point $\mathrm{z}_{0}$ in it as the initial point of a trajectory. Then, on the basis of Liouville's theorem on the conservation of phase space volume [2], one can prove that after a sufficiently long time the system will return to the same area A (except, possibly, for a set of initial points of measure zero). From that a stronger statement follows: a closed Hamiltonian system in a finite volume will return infinitely many times to the same area. These returns are known as the Poincaré cycles. The intervals between them can obey any law and be even stochastic, but as E. Zermelo pointed out in 1896, Poincaré's recurrence theorem and Boltzmann's H-theorem are absolutely incompatible. Then how the irreversible Boltzmann equation (29) can be obtained from the reversible Hamilton system (12) with the use of the BBGKY hierarchy?

The chain of equations (21) containing $\mathrm{N}$ equations for the functions $\mathrm{F}_{\mathrm{s}}(\mathrm{s}=1, \ldots, \mathrm{N})$, is completely equivalent to one Liouville's equation (14) for the function $\mathrm{F}_{\mathrm{N}}$, thus being equivalent to a set of Newton equations for $\mathrm{N}$ particles. So, it does not lead to any effect of irreversibility in time. If, for example, one breaks the hierarchy of the BBGKY equations limiting oneself to the two equations (23) and (24), the effect of irreversibility will by no means appear. As we mentioned above, the irreversibility appears in the equations (23), (24) as a result of the use of the non-mechanical postulate of factorization (26) for all distribution functions $\mathrm{F}_{\mathrm{s}}(\mathrm{s} \geq 2)$ at $\mathrm{t}=-\infty$.

G. A. Martynov [5] noted that the derivation of the hierarchy of BBGKY equations does not take into account the non-stability of solutions of Newton's equations for a system of molecules, whose surface curvature is always negative $[6,7]$. After 7-10 collisions, the uncertainty of the angular distribution of colliding molecules becomes more than $2 \pi$ and, as a result, an unambiguous calculation of particle trajectories becomes principally impossible. Then there remains only one possibility: to describe the system by methods of probability theory, that means to proceed to the Gibbs ensemble, global probability $\mathrm{F}_{\mathrm{N}}$, and the Liouville equation for the determination of $\mathrm{F}_{\mathrm{N}}$. However, since after 7-10 collisions a stochastization of trajectories occurs, it is useless, in the hierarchy of distribution functions (16) - (18), to take into account the terms with the index s bigger than some limit value $\mathrm{s}^{*}$. That is, only several dozen (hundred) of particles determine the state of the substance in a given point; for the rest of the particles one can pass to the thermodynamic limit:

$$
N \rightarrow \infty, \quad V \rightarrow \infty, \quad \frac{N}{V}=n_{0}=\text { const }
$$

Thus, the hierarchy of BBGKY equations includes a parameter $\left(\mathrm{n}_{0}\right)$, which will be known only after the thermodynamic equilibrium is established. And that violates the principle of causality, since the solutions of the BBGKY equations that have physical meaning can only depend on the parameters defined at the time $t^{\prime} \leq t$ and in no way can depend on $n_{0}=n(\vec{r}, t=\infty)$.

In order to obtain somehow physically sensible equations, G. A. Martynov [5] proposed to expand the BBGKY hierarchy equations in an appropriate small parameter.

So, the state of a substance in a given point is defined actually by several dozen (hundred) of molecules limited by a

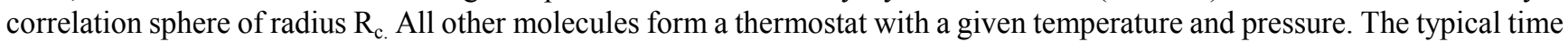
of relaxation inside this sphere is equal to $t_{\mathrm{c}}$. For the entire isolated macroscopic system under consideration we introduce two global parameters: the system size $\mathrm{L}$ and the typical relaxation time $\mathrm{t}_{\mathrm{r}}$. Now one can introduce the following two dimensionless parameters into the theory:

$$
\varepsilon=\frac{R_{c}}{L}, \quad \chi=\frac{t_{c}}{t_{r}} .
$$

The simplest estimates show that for the majority of macroscopic systems 


$$
R_{c}<<L, t_{c}<<t_{r}
$$

so the parameters (31) are extremely small (about $10^{-8}$ and less).

All hydrodynamic variables of macro-systems change significantly only on the distances of the order $L$ and on the time intervals of the order $t_{r}$. Therefore they are functions of the dimensionless distance $r / L$ and dimensionless time $t / t_{r}$ :

$$
\rho=\rho\left(\frac{\vec{r}}{L}, \frac{t}{t_{r}}\right), \quad v_{0, i}=v_{0, i}\left(\frac{\vec{r}}{L}, \frac{t}{t_{r}}\right), \quad T=T\left(\frac{\vec{r}}{L}, \frac{t}{t_{r}}\right) .
$$

The hydrodynamic variables are connected with the one-particle distribution function $\mathrm{F}_{1}\left(\mathrm{t}, \overrightarrow{\mathrm{r}}_{\mathrm{i}}, \overrightarrow{\mathrm{p}}_{\mathrm{i}}\right)$, so they depend on the same dimensionless variables (33), as well as on the dimensionless momentum $p / \mathrm{P}$ :

$$
F_{1}\left(\frac{\vec{r}}{L}, \frac{\vec{p}}{\mathrm{P}}, \frac{t}{t_{r}}\right)=\exp \left\{\omega_{(1)}\left(\frac{\vec{r}}{L}, \frac{\vec{p}}{\mathrm{P}}, \frac{t}{t_{r}}\right)\right\} .
$$

The function $\mathrm{F}_{1}\left(\mathrm{t}, \overrightarrow{\mathrm{r}}_{\mathrm{i}}, \overrightarrow{\mathrm{p}}_{\mathrm{i}}\right)$ together with the hydrodynamic variables forms a group of "slow functions". Using the boundary condition of relaxation of correlations (26), one gets for multi-particle distribution functions

$$
F_{1, \ldots, s^{*}}=\exp \left(\Omega_{1, \ldots, s^{*}}\right) \prod_{i=1}^{s^{*}} F_{i}, \quad\left(\Omega_{1} \equiv 0\right)
$$

where $\Omega_{1, \ldots, s^{*}}=\Omega_{\left(s^{*}\right)}\left(\frac{\overrightarrow{r_{1}}}{R_{c}}, \ldots, \frac{\overrightarrow{r_{s}}}{R_{c}} ; \frac{\overrightarrow{p_{1}}}{P}, \ldots, \frac{\overrightarrow{p_{s^{*}}}}{\mathrm{P}} ; \frac{\mathrm{t}}{\mathrm{t}_{\mathrm{c}}}\right) \rightarrow 0$ as $\mathrm{r}_{\mathrm{g}} \rightarrow \infty$.

The functions $\Omega_{\left(\mathrm{s}^{*}\right)}$ introduced in such way describe a mutual correlation of particles inside the correlation sphere. They form a group of "fast" functions, which can vary essentially in time intervals $t \approx t_{\mathrm{c}}$ and on distances about $R_{\mathrm{c}}$, but they equal zero outside the correlation sphere.

Due to the inequality (32), all slow functions are quasi constant on the distances of the order of $R_{\mathrm{c}}$. Therefore, the functions $\mathrm{F}_{1}$ and (33) at the solution of equations for $\Omega_{\left(\mathrm{s}^{*}\right)}$ may be considered constant and not dependent on $\mathrm{r}$.

After substitution (34) and (35) into the BBGKY hierarchy equations (21), one again obtains a hierarchy of equations for

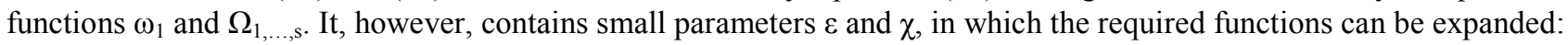

$$
\begin{gathered}
\omega_{1}=\omega_{1}^{(0)}+\varepsilon \cdot \omega_{1}^{(1)}+\ldots, \\
F_{1}=F_{1}^{(0)}+\varepsilon \cdot F_{1}^{(1)}+\ldots=F_{1}^{(0)}\left(1+\varepsilon \cdot \omega_{1}^{(1)}+\ldots\right),(\varepsilon=\chi) \\
\Omega_{1, \ldots, s^{*}}=\Omega_{1, \ldots, s^{*}}^{(0)}+\varepsilon \cdot \Omega_{1, \ldots, s^{*}}^{(1)}+\ldots,
\end{gathered}
$$

Then one can follow the standard procedure of the perturbation theory. For the sake of simplicity, in (36) and later on $\varepsilon=\chi$.

The goal of the expansion of the BBGKY hierarchy equations in the small parameter $\varepsilon$ is not only (and not so much) to make them simpler, but rather to individuate a group of equations describing real physical processes. Therefore G. A. Martynov indicates in [5] that, for the development of the BBGKY theory, such expansion is a step as principal as the transition from the Liouville equation to the BBGKY hierarchy. In the first order in $\varepsilon$, one gets from (36) an equation for $\omega_{1}{ }^{(1)}$ :

$$
\frac{\partial \omega_{1}^{(0)}}{\partial t}+\frac{\overrightarrow{p_{1}}}{m} \frac{\partial \omega_{1}^{(0)}}{\partial \overrightarrow{r_{1}}}=-\overrightarrow{f_{(1)}^{(1)}} \frac{\partial \omega_{1}^{(0)}}{\partial \overrightarrow{p_{1}}}+\eta_{(1)}^{(1)}
$$

where $\overrightarrow{f_{(1)}^{(1)}}$ is the force acting on a particle from all other particles, $\eta_{(1)}^{(1)}$ is the source of the correlation entropy. Using the Enskog-Chapman method, the function $\omega_{1}{ }^{(1)}$ can be presented in the range of small deflections from equilibrium as

$$
\omega^{(0)}=\ln \frac{n(\vec{r}, t)}{n_{0}}-\frac{\left[\vec{p}-m\langle\vec{v}\rangle_{\vec{r}, t}\right]^{2}}{2 m T(\vec{r}, t)} .
$$

Substituting this expression in (37) and using Euler's hydrodynamic equations [1], for "slow" variables one gets: 


$$
\begin{aligned}
& \frac{\partial \omega_{1}^{(0)}}{\partial t}+\frac{\overrightarrow{p_{1}}}{m} \frac{\partial \omega_{1}^{(0)}}{\partial r_{1}}=\left\{1-\frac{1}{T}\left(\frac{\partial \mathrm{P}^{(0)}}{\partial n}\right)_{T}\right\} \frac{p^{*}{ }_{\alpha}}{m} \frac{1}{n} \frac{\partial n}{\partial r_{\alpha}}+\left\{\frac{p^{* 2}}{2 m T}-\frac{1}{n}\left(\frac{\partial \mathrm{P}^{(0)}}{\partial T}\right)_{n}\right\} \frac{p^{*}{ }_{\alpha}}{m} \frac{1}{T} \frac{\partial T}{\partial r_{\alpha}}+ \\
& +\frac{p^{*}{ }_{\alpha} p^{*}{ }_{\beta}}{m T} D_{\alpha \beta}+\left(\frac{p^{* 2}}{m T}-1\right) \frac{1}{3} \frac{\partial\left\langle v_{\alpha}\right\rangle}{\partial r_{\alpha}}=\sum_{k=1}^{4} X^{(k)} x^{(k)}
\end{aligned}
$$

where $\overrightarrow{p^{*}}=\vec{p}-m\langle\vec{v}\rangle, \quad D_{\alpha \beta}=\frac{1}{2}\left(\frac{\partial\langle v\rangle_{\alpha}}{\partial r_{\beta}}+\frac{\partial\langle v\rangle_{\beta}}{\partial r_{\alpha}}\right)$ is the tensor of velocity deformation, $x^{(k)}$ are gradients of hydrodynamic variables

$$
x^{(1)}=\frac{\partial n}{\partial \vec{r}}, \quad x^{(2)}=\frac{\partial T}{\partial \vec{r}}, \quad x_{\alpha \beta}^{(3)}=D_{\alpha \beta}, \quad x^{(4)}=\frac{\partial\left\langle v_{\alpha}\right\rangle}{\partial r_{\alpha}}
$$

and $X^{(k)}$ are the factors corresponding to these variables in (39).

In order to obtain the transport equation of the first order in $\varepsilon$, one should use the exact balance equations for the number of particles, momentum, and temperature [1]. The derivatives of hydrodynamic variables in the left part of these equations do not need to be expanded into series, because they are equal to their exact values, while the right parts containing the strain tensor $P_{i k}$ and the heat flux q should be expanded:

$$
\begin{gathered}
P_{\alpha \beta}=\delta_{\alpha \beta} P^{(0)}-\lambda_{\alpha}^{(n)} \frac{\partial n}{\partial r_{\beta}}-\lambda_{\alpha}^{(T)} \frac{\partial T}{\partial r_{\beta}}-\lambda^{(D)} D_{\alpha \beta}-\lambda_{\alpha \beta}^{(v)} \frac{\partial\left\langle v_{\gamma}\right\rangle}{\partial r_{\gamma}} \\
q_{\alpha}=-\mu^{(n)} \frac{\partial n}{\partial r_{\alpha}}-\mu^{(T)} \frac{\partial T}{\partial r_{\alpha}}-\mu_{\beta}^{(D)} D_{\alpha \beta}-\mu_{\alpha}^{(v)} \frac{\partial\left\langle v_{\gamma}\right\rangle}{\partial r_{\gamma}}
\end{gathered}
$$

In the case of multi-component systems one should add to (40) analogous formulas for mass fluxes of each component. The substitution of (40) into the balance equation gives a generalized system of Navier-Fourier-Stokes drift equations valid within the accuracy up to the order of $\varepsilon^{2}$ :

$$
\begin{gathered}
\frac{\partial n}{\partial t}-\frac{\partial\left(n\left\langle v_{\alpha}\right\rangle\right)}{\partial r_{\alpha}}=0 \\
n \frac{d\left(m\left\langle v_{\alpha}\right\rangle\right)}{d t}+\frac{\partial P^{(0)}}{\partial r_{\alpha}}=\frac{\partial}{\partial r_{\beta}}\left(\lambda_{\alpha}^{(n)} \frac{\partial n}{\partial r_{\beta}}\right)+\frac{\partial}{\partial r_{\beta}}\left(\lambda_{\alpha}^{(T)} \frac{\partial T}{\partial r_{\beta}}\right)+\frac{\partial\left(\lambda^{(D)} D_{\alpha \beta}\right)}{\partial r_{\beta}}+\frac{\partial}{\partial r_{\beta}}\left(\lambda_{\alpha \beta}^{(v)} \frac{\partial\left\langle v_{\gamma}\right\rangle}{\partial r_{\gamma}}\right) \\
n \frac{d\left(\frac{3}{2} T\right)}{d t}=\frac{\partial}{\partial r_{\alpha}}\left(\mu^{(n)} \frac{\partial n}{\partial r_{\alpha}}\right)+\frac{\partial}{\partial r_{\alpha}}\left(\mu^{(T)} \frac{\partial T}{\partial r_{\alpha}}\right)+\frac{\partial}{\partial r_{\alpha}}\left(\mu_{\beta}^{(D)} D_{\alpha \beta}\right)+\frac{\partial}{\partial r_{\alpha}}\left(\mu_{\alpha}^{(v)} \frac{\partial\left\langle v_{\gamma}\right\rangle}{\partial r_{\gamma}}\right) .
\end{gathered}
$$

In the case of multi-component systems, these equations should be completed by equations of mass conservation of each component analogous in structure to the equation (43).

The equations (41)-(42) form a closed system of equations to determine the five required hydrodynamic variables $\mathrm{n}$, $\left\langle v_{\alpha}\right\rangle$, T. They are conservation laws for the number of particles, momentum, and temperature, respectively.

Unlike the ordinary Navier-Stokes or Fourier equations, the equations (41)-(43) contain terms with gradients of all hydrodynamic variables, i.e. describe the "cross" phenomena neglected in ordinary transport equations. They are the basis of I. Prigogine's non-equilibrium thermodynamics in the range of small (linear) deflections from equilibrium. To obtain the transport equations for larger deflections, one should use more and more exact expansions in the parameter $\varepsilon$ : at first, within the accuracy of up to $\varepsilon^{3}$, then up to $\varepsilon^{4}$, etc.

Unlike $\mathrm{n},\left\langle v_{\alpha}\right\rangle$, and $\mathrm{T}$, entropy is not a conservative hydrodynamic variable. The reason is that the correlation sphere defining all the local parameters of the substance is an open system continuously exchanging particles, momentum, and energy with the surrounding "thermostat". At that, the local entropy $s(r, t)$ (unlike the global entropy of the entirely closed system of $\mathrm{N}$ particles) should not always increase. Thus, the global increase of chaos in an entirely isolated system might be 
accompanied by a local increase of order, for example, by the appearance of crystal grains in an overcooled liquid or by the formation of biological structures in solutions.

However, the mechanical entropy of an isolated system of $\mathrm{N}$ particles must be constant as well as the total momentum and energy of the system. And all the constructions discussed above by no means abolish Poincaré's theorem. Then how one can reconcile that with the well-known thermodynamic law of global entropy increasing? To clarify this question, G. A. Martynov [5] used the idea of the local correlation entropy

$$
S^{(l)}(t)=\int_{V} n(\vec{r}, t) s^{(l)}(\vec{r}, t) d^{3} r .
$$

Since the entropy $S^{(l)}$ is proportional to the integral over $\omega_{(l)}$, then, at $l \geq \mathrm{s}^{*}$ ( $\mathrm{s} *$ is the maximal number of particles in the correlation sphere), $S^{(l)}$ becomes equal zero because $\omega_{(l)}=0$. Hence

$$
\frac{d S^{(l)}}{d t}=J^{(l-1)}-J^{(l)}=0, \quad \text { at } \quad 1 \geq \mathrm{s}^{*},
$$

where $J^{(l)}=\int \frac{\partial \Phi_{1, l+1}}{\partial \overrightarrow{r_{1}}} \frac{\partial \omega_{1, \ldots, l+1}}{\partial \overrightarrow{p_{1}}} F_{1, \ldots, l+1} d(1) \ldots d(l+1)$.

As $J^{(\mathbb{N})}=0$, the equality (45) is valid for all $l \geq \mathrm{s}^{*}$, except for $l=\mathrm{N}$ :

$$
\frac{d S^{(N)}}{d t} \geq 0 .
$$

The entropy originates in the correlation sphere, i.e. for $l \leq \mathrm{s}^{*}$. The N-particle correlation serves as an entropy sink and all the intermediate correlations with $\mathrm{s}^{*}<l<\mathrm{N}-1$ play the role of entropy conductors that transport it without loss from the source to the sink. The source power is defined by what takes place inside the correlation sphere, whereas the outflow power is determined by the reaction of the entire system. At a transition to the thermodynamic limit, the outflow goes off to infinity and disappears, so the global entropy per one particle should not be constant any more. Thus the main obstacle on the way of mechanical justification of thermodynamic laws is removed.

However, this mechanical justification of thermodynamics was realized in [5] at the cost of non-constructive (in terms of metamathematics) operations: a transition to the thermodynamic limit and going off to infinity. For the "ordinary" XIX century set-theoretic mathematics the going to infinity is an unquestionable procedure. However, in the constructive mathematical analysis of the XX century the abstraction of actual infinity is absent. Roughly, one can justify "whatever you like" by a non-constructive (i.e. non-formalizable and non-finite) operation of going to infinity. It is indicative that in [5] there was successfully proved the variability of the global dynamic entropy, but not its nondecreasing character (see (11)). G. A. Martynov in [5] confined himself only to an optimistic statement that "the further research seems unlikely not to remove the contradiction between the Newton mechanics and thermodynamics". However, even such highly experienced theoreticians as L. D. Landau and E. M. Lifschitz stated that "it is unclear, whether the law of entropy increase can be in general obtained from the classical mechanics" [8].

One can admit that G. A. Martynov managed to deduce the thermodynamics from the Liouville equation (i.e. from Newton's mechanics), if one closes his eyes to the fact that he has used a non-constructive operation of going to infinity. But G. A. Martynov did not even try to obtain the L. Boltzmann kinetic theory from mechanics, because he believed that N. N. Bogolubov's deduction of the equations (26)-(29) was incorrect [5]. The point is that in Bogolubov's method only first two terms of the series converge in $n_{0}$, while all other terms diverge. The cause of that is just the presence of the parameter $n_{0}=n(\vec{r}, t=\infty)$ violating the causality principle [5]. As a result, the expansion in terms $\mathrm{n}_{0}$ leads to non-physical results.

G. A. Martynov also believes one other method used by N. N. Bogolubov to be incorrect. We remind that the collision integral in the right part of the equation (1) contains one-particle distribution functions defined at times $t-\tau$ and $t+\tau$, i.e. before and after collision, so a transition to the limit $\tau \rightarrow 0$ is impossible, because the very idea of collision becomes meaningless. Meanwhile, all the distribution functions $\mathrm{F}_{\mathrm{s}}$ in the BBGKY hierarchy are defined at one and the same time $t$, so the idea of trajectory is excluded at all. In other words, Boltzmann's theory and the BBGKY hierarchy are incompatible, and, according to Martynov, Bogolubov's technique of introducing trajectory into the BBGKY hierarchy with the use of the displacement operator (28) is not deductive, because this operator introduces into the hierarchy a new time $t-\tau$, which was not there. As a result, the Boltzmann kinetic theory cannot be considered as a limiting case of the BBGKY hierarchy valid at $\rho \rightarrow 0$. (We do agree with this conclusion). However, as G. A. Martynov notes, from that it does not follow that Boltzmann's theory is erroneous. It has the right to exist independently as an efficient approximation theory. For example, the van der Waals state equation is one of the best state equations, although it also cannot be derived from exact equations of statistical mechanics. In that we not only agree with G. A. Martynov, but are of a more radical opinion on which equation (that of 
Boltzmann or that of Liouville and BBGKY) is approximate and which is exact. That question will be discussed in Section 6.

\section{The Determinism of Newton Mechanics. The Theorems by Poincaré, Khinchin, and Others}

The mathematical formalism of classical dynamics is the theory of differential equations (DE) both ordinary and in partial derivatives. According to Birkhoff [9], to describe classical dynamical systems one should consider a system of DE

$$
\frac{d x_{i}}{d t}=X_{i}\left(x_{1}, x_{2}, \ldots, x_{n}\right), \quad(i=1,2, \ldots, n)
$$

where the right parts are continuous functions of point $p\left(x_{1}, x_{2}, \ldots, x_{n}\right)$ in a closed domain $\mathrm{D}$ of an $n$-dimensional Euclidean phase space. At that, it is supposed that these functions satisfy an additional condition (the Lipschitz condition), which provides the uniqueness of the solution defined by the initial data

$$
x_{1}=x_{1}{ }^{(0)}, x_{2}=x_{2}{ }^{(0)}, \ldots, x_{n}=x_{n}{ }^{(0)}, \text { at } t=0,
$$

where $p_{0}\left(x_{1}^{(0)}, x_{2}{ }^{(0)}, \ldots, x_{n}^{(0)}\right)$ is the initial point of motion. For DE (47) with the condition (48), a number of general properties of motion is proved. Thus, the Cauchy theorem states [10]:

Let a set of ordinary differential equations be given:

$$
\frac{d y_{k}}{d x}=f_{k}\left(x, y_{1}, y_{2}, \ldots, y_{n}\right)=\sum_{m_{0}, \ldots, m_{n}=0}^{\infty} a_{m_{0} m_{1} \ldots m_{n}}^{(k)}\left(x-x_{0}\right)^{m_{0}}\left(y_{1}-y_{1}^{0}\right)^{m_{1}} \ldots\left(y_{n}-y_{n}^{0}\right)^{m_{n}}, \quad(k=1, \ldots, n)
$$

where the series of the right part converge in the domain $\left|x-x_{0}\right|<\rho,\left|y_{k}-y_{k}^{0}\right|<r, k=1, \ldots, n$.

Then there exists a unique and, at that, continuous solution of the system (49) with the initial conditions

$$
y_{k}\left(x_{0}\right)=y_{k}^{0}(k=1, \ldots, n) \text {. }
$$

If the Lipschitz condition is satisfied, a stronger result is obtained, namely Picard's theorem:

Let the system of DE

$$
\frac{d y_{k}}{d x}=f_{k}\left(x, y_{1}, y_{2}\right),(k=1,2)
$$

be defined in the domain $\left|x-x_{0}\right| \leq a,\left|y_{k}-y_{k}^{0}\right| \leq b,(k=1,2)$, and $f_{1}$ and $f_{2}$ be continuous in this domain, thus $\left|\mathrm{f}_{\mathrm{k}}\right| \leq \mathrm{M}=$ const. Then, if $f_{1}$ and $f_{2}$ satisfy in this domain the Lipschitz condition in $y_{1}$ and $y_{2}$

$$
\left|f_{k}\left(x, y^{*}, y^{*}{ }_{2}\right)-f_{k}\left(x, y_{1}, y_{2}\right)\right| \leq L\left(\left|y^{*}-y_{1}\right|+\left|y^{*}{ }_{2}-y_{2}\right|\right),(k=1,2)
$$

where $L=$ const $>0$, and $x, y_{1}, y_{2}, y^{*}, y^{*}{ }_{2}$ are arbitrary, then there exists a solution of the system (51), which satisfies the condition

$$
y_{k}(x) \rightarrow y_{k}^{0} \text { at } x \rightarrow x_{0},(k=1,2)
$$

At that, this solution is continuous, differentiable, unique, and exists at least in the domain $\left|x-x_{0}\right| \leq h=\min \left(a, \frac{b}{M}\right)$.

The theorem on the continuous dependence on parameters is also of importance:

Let a system of DE be given:

$$
\left.\begin{array}{c}
\frac{d y_{1}}{d x}=f_{1}\left(x, y_{1}, \ldots, y_{n}, \lambda_{1}, \ldots, \lambda_{m}\right), \\
---------------- \\
\frac{d y_{n}}{d x}=f_{n}\left(x, y_{1}, \ldots, y_{n}, \lambda_{1}, \ldots, \lambda_{m}\right),
\end{array}\right\}
$$

where $f_{1}, \ldots, f_{n}$ are defined in $x, y_{1}, \ldots, y_{n}$ in the domain $\left|x-x_{0}\right| \leq a,\left|y_{k}-y_{k}{ }^{0}\right| \leq b,(k=1, \ldots, n)$ and in $\lambda_{1}, \ldots, \lambda_{m}$ in the domain $\left|\lambda_{k}\right| \leq \lambda_{k}^{*}(k=1, \ldots, n)$. Then, let $f_{k}$ be continuous in all their variables in these domains and, consequently, limited, i.e. $\mid f_{k}\left(x, y_{1}\right.$, $\lambda) \mid \leq M=$ const for $(x, y)$ and $\lambda$ belonging to the indicated domains. Then, let $f_{k}(k=1, \ldots, n)$ satisfy the Lipschitz condition in $y_{1}, \ldots, y_{n}$ in the same domains:

$$
\left|f_{k}\left(x, y^{*}, \lambda\right)-f_{k}\left(x, y^{* *}, \lambda\right)\right| \leq L \sum_{\mathrm{k}=1}^{\mathrm{n}}\left|y_{k}^{*}-y_{k}^{* *}\right|
$$


where $L$ does not depend on $(x, y, \lambda)$. Then the system (53) has a unique solution

$$
y_{k}=y_{k}\left(x, \lambda_{1}, \ldots, \lambda_{m}\right),(k=1, \ldots, n)
$$

that satisfies the initial conditions

$$
y_{k}\left(x_{0}\right)=y_{k}^{0} .(k=1, \ldots, n)
$$

This solution is defined and differentiable continuously as a function of $x$ in the interval $\left|x-x_{0}\right| \leq h=\min \left(a, \frac{b}{M}\right)$. Moreover, the solution (54) is uniformly continuous in the indicated intervals in $\lambda_{1}, \ldots, \lambda_{m}$, i.e. $\left|y_{k}(x, \lambda)-y_{k}\left(x, \lambda^{*}\right)\right| \leq \varepsilon$, where for any $\varepsilon$ such $\delta$ can be found that $\left|\lambda_{k}-\lambda_{k}^{*}\right| \leq \delta(k=1, \ldots, n)$.

If the right part of DE is continuous (which just takes place for the dynamical systems (47)), then Peano's theorem is valid: Let a differential equation be given

$$
\frac{d y}{d x}=f(x, y),
$$

Let $\mathrm{f}(\mathrm{x}, \mathrm{y})$ be defined in the domain $\left|x-x_{0}\right| \leq a,\left|y-y_{0}\right| \leq b$, where $a$ and $b$ are given positive numbers. If the function $f(x, y)$ is continuous and, hence, limited in R (i.e. there exists a positive number $M$ such that the inequality $f(x, y) \leq M$ is valid for all points $(x, y)$ of $\mathrm{R})$, then the equation (56) has at least one solution

$$
y=\varphi(x),
$$

which satisfies the initial conditions

$$
y=y_{0} \text { at } x=x_{0} .
$$

This solution is defined and continuous together with the first derivative in the interval

$\left|\mathrm{x}-\mathrm{x}_{0}\right| \leq \mathrm{h}, \quad$ where $\mathrm{h}=\min \left(\mathrm{a}, \frac{\mathrm{b}}{\mathrm{M}}\right)$.

The theorems (49)-(58) allow one to individuate a number of general properties of the motions defined by the system (47):

A. Any solution can be either continued infinitely as $t \rightarrow \pm \infty$, or it reaches the boundary of the domain $D$ at a finite value $t=t^{*}$.

B. Any solution to the system (47)

$$
x_{i}=f_{i}\left(t, x_{1}{ }^{(0)}, \ldots, x_{n}{ }^{(0)}\right),(i=1,2, \ldots, n)
$$

is a continuous function of time $t$ and coordinates of the initial point.

C. Since the right parts of (47) do not depend on time, then, if the motion starting at the time $t$ from the point $p$ reaches the point $p_{1}$, and the motion starting at the time $t_{2}$ from the point $p_{1}$ reaches the point $p_{2}$, then the first motion reaches the point $p_{2}$ at the time $t_{1}+t_{2}$ ( group property).

The universal properties (A, B and C) of dynamical systems listed above express the MECHANICAL DETERMINISM: one can anyhow increase the time $t$, but the solution of the dynamical problem (47) will remain single-valued. On the contrary, one can go along the trajectory in the opposite direction as $\mathrm{t} \rightarrow-\infty$ and completely restore the history of the motion. The inevitability and uniqueness of solutions to the dynamical equations (47) is such that it allowed P. Laplace to declare: "Give me the initial conditions and I will predict the future of the Universe". It is just after the works of P. Laplace that the determinism of Newton's mechanics was extended over all natural phenomena and became known as the Laplacian determinism.

Under the influence of "a truly triumphal march of mechanics" [11] with its determinism and theorems of uniqueness, it is believed up to now to be a matter of honor to make any science perfect bringing it to the level of mechanical exactness, i.e. making it completely deterministic. "The principle of determinism serves as a guiding principle in all the fields of scientific knowledge, an effective tool for comprehension of truth" [12]. In materialistic philosophy, deviations from determinism were equated with the negation of causality, while non-deterministic theories of catastrophes were condemned as "pushing through the idea of God" [13]. On the contrary, the adherents of indeterminism used to accuse the advocates of determinism in fatalism [14].

Let us observe the problem of determinism and causality in classical mechanics from a modern perspective. Looking at the theorems of Cauchy, Picard, and Peano, it is hardly possible to reject the idea of fatalism. True, now we know a class of DE with branching solutions [15].

Consider, for instance, the system of DE in a vector form

$$
\frac{d x}{d t}=f(t, x)+\lambda g(t, x, \lambda),
$$


where $x \equiv\left(x_{1}, x_{2}, \ldots, x_{n}\right)$ is the required vector of space $E_{n}, f \equiv\left(f_{1}, f_{2}, \ldots, f_{n}\right)$ and $g \equiv\left(g_{1}, g_{2}, \ldots, g_{n}\right)$ are given vector-functions valued in $E_{n}$ continuous in the set of variables $(t, x, \lambda) \in(-\infty,+\infty) \times \mathrm{G} \times \Lambda$ and $\omega$ - periodic in $t$. It is supposed that $f$ is holomorphic in $x$, and $g$ is holomorphic in $(x, \lambda) \in \mathrm{G} \times \Lambda$. Let $\varphi(t) \equiv\left(\varphi_{1}(t), \ldots, \varphi_{n}(t)\right) \subset \mathrm{G}$ be any $\omega$-periodic solution of the generating system of DE

$$
\frac{d y}{d t}=f(t, y)
$$

Then one can formulate the following problem (Poincaré's problem): to find, at sufficiently small $|\lambda|$, all continuous and $\omega-$ periodic solutions $\psi(t, \lambda)$ of the system (59) satisfying the condition

$$
\psi(t, 0)=\varphi(t) .
$$

Theorems have been proved [15] that the equations (59) and (60) have branching solutions. But the right part of (59) depends on time $t$, and in the equations (47) the right parts are only functions of the point $p\left(x_{1}, x_{2}, \ldots, x_{n}\right)$ of phase space. That is why, no loophole can be seen so far to violate the determinism and uniqueness of solutions of the dynamical equations (47). May be, that is possible under the conditions of instability of solutions (in the sense of Lyapounov)?

The stability theory for solutions of DE was developed by A. Poincaré, A. M. Lyapounov and others [9, 10, 16-18]. Let M be a dynamical system on a locally compact metrical space R. A motion is called positively (negatively) Lagrange stable, if its half-trajectory $\mathrm{f}(\mathrm{p} ; 0,+\infty)(\mathrm{f}(\mathrm{p} ; 0,-\infty))$ is contained in a compact set of space $\mathrm{R}$.

The system (49) can be rewritten in a more compact vector form:

$$
\frac{d \mathbf{y}}{d t}=f(t, \mathbf{y})
$$

Let us single out a motion $y=\varphi(t)$ of the system (61) and call it non-perturbative. The motion $y=\varphi(t)$ is Lyapunov stable, if for each $\varepsilon>0$ one can find such $\delta>0$ that at $t>\mathrm{t}_{0}$ from $\left\|\mathrm{y}\left(\mathrm{t}_{0}\right)-\varphi\left(\mathrm{t}_{0}\right)\right\|<\delta$ it follows $\|\mathrm{y}(\mathrm{t})-\varphi(\mathrm{t})\|<\varepsilon$. Here $\mathrm{y}(\mathrm{t})$ means any other solution of the DE system (61), defined by the initial conditions $y=y\left(t_{0}\right)$ at $t=t_{0}$.

A motion is called asymptotically Lyapounov stable, if it is Lyapounov stable and, besides, there exists such number $\mathrm{h}>0$ that

$$
\lim _{\mathrm{t} \rightarrow \infty}\|\mathbf{y}(\mathrm{t})-\varphi(\mathrm{t})\|=0, \quad \text { if }\left\|\mathbf{y}\left(\mathrm{t}_{0}\right)-\varphi\left(\mathrm{t}_{0}\right)\right\|<\mathrm{h} .
$$

If the condition (62) is valid uniformly in $\mathrm{t}_{0}$, then the asymptotic stability is called uniform in $\mathrm{t}_{0}$.

For an autonomous system like (47) the asymptotic stability is always uniform in initial data. The point $p$ is called positively Poisson stable ( $\mathrm{P}^{+}$-stable), if for any vicinity $\mathrm{U}$ of the point $\mathrm{p}$ and for any $\mathrm{T}>0$ there exists such $\mathrm{t} \geq \mathrm{T}$ that $\varphi(t, p) \in$ $U$. Analogously, if there exists such $t \leq-T$ that $\varphi(t, p) \in U$, then the point $p$ is negatively Poisson stable $\left(\mathrm{P}^{-}-\right.$stable) . If the point is simultaneously $\mathrm{P}^{+}$- and $\mathrm{P}^{-}$-stable, then it is Poisson stable (P-stable). An invariant closed set $\mathrm{V}$ is called the attraction center of the motion $\varphi(t, p)$ as $\mathrm{t} \rightarrow+\infty(-\infty)$, if the probability of the point $\mathrm{p}$ to stay in $\mathrm{S}(\mathrm{V}, \varepsilon)$ is equal to unity for any $\varepsilon>0$ :

$$
\mathrm{P}(\varphi(t, p)) \in \mathrm{S}(\mathrm{V}, \varepsilon)=1 \text { (for any } \varepsilon>0) .
$$

If the set $\mathrm{V}$ does not contain any other attraction center, then $\mathrm{V}$ is called the minimal attraction center.

Theorem I [9]: If the motion $\varphi(t, p)$ is positively/negatively Lagrange stable, then for it there exists a minimal attraction center as $t \rightarrow+\infty(-\infty)$.

Let us consider a linear system of DE

$$
\frac{d y}{d t}=A(t) y+f(t)
$$

where $A(t)$ and $f(t)$ are continuous in $t(n \times n)$ and $(n \times 1)$ matrices with a common period $\omega>0$.

Theorem II [18]: Let a homogeneous system of DE (64) has at least $k$ linearly independent $\omega$-periodic solutions $\varphi_{1}(t), \ldots, \varphi_{k}(t)$ $(1 \leq k \leq n)$. Then a non-homogeneous system (64) has a unique $\omega$-periodic solution if and only if the conditions of orthogonality

$$
\int_{0}^{\omega}\left(\psi_{s}(t), f(t)\right) d t=0, \quad(s=1, \ldots, k)
$$

are satisfied. Here $\psi_{i}(t)(i=1, \ldots, k)$ are the linearly independent $\omega$-periodic solutions of the adjoint system

$$
\frac{d \xi}{d t}=-A *(t) \xi
$$


Theorem III (Masser) [18]: If the system (64) has a bounded solution $y(t)(t \geq 0)$, then a $\omega$-periodic solution to this system exists.

Corollary: if the system of DE (64) has no $\omega$-periodic solutions, then all their solutions are unbounded on both half-axes ( $t \geq 0$ and $\mathrm{t} \leq 0)$.

These theorems are given to illustrate the fact that even unbounded solutions of DE are unique. As for bounded solutions, they are characterized by an unavoidable determinism. The "recurrence theorems" are an extreme form of such determinism [9]:

Theorem IV (Poincaré -Carathéodory recurrence theorem for sets). Let in the phase space R of the dynamical system (61) there exist an invariant measure $\mu$ such that $\mu \mathrm{R}=1$. And let $\mathrm{A} \subset \mathrm{R}$ be a measurable set of measure $\mathrm{m}(\mu \mathrm{A}=\mathrm{m}>0)$. Then there exist such values $\mathrm{t}(|\mathrm{t}| \geq 1)$ that $\mu[\mathrm{A} \cdot \varphi(\mathrm{t}, \mathrm{A})]>0$.

Theorem V (Poincaré -Carathéodory recurrence theorem for points). If the phase space $\mathrm{R}$ of the dynamical system $\varphi(t, p)$ has a countable base and an invariant measure $\mu$ such that $\mu R=1$ exists in $R$, then almost all points $p \in R$ (in the sense of measure $\mu$ ) are Poisson stable, i.e. for the set $\mathrm{E}^{*}$ of Poisson unstable points one has $\mu \mathrm{E}^{*}=0$.

1. The Poincaré recurrence theorems state that a system of finite energy contained in a finite spatial volume will, after a sufficiently long time, return to an arbitrarily small neighborhood of its initial state. It should be noted that "a sufficiently long time" could be much longer than the predicted lifetime of the Universe.

Theorem IV states only that for any set $\mathrm{E}(\mu \mathrm{E}>0)$, there exist such arbitrary large absolute values $t$ that $\mu(\mathrm{E} \cdot \varphi(t, \mathrm{E}))>0$. However, theorem IV does not supply any estimate of the upper limit of the measure of intersection. Furthermore, the "physical consequences" of the Poincaré-Carathéodory theorems are not evident. The Khinchin theorem [9] obviates these drawbacks.

Theorem VI (Khinchin): under the conditions of theorem IV, for any measurable set $\mathrm{E}(\mu \mathrm{E}=m>0)$ and any $t(-\infty<t<+\infty)$ the inequality

$$
\mu(t)=\mu(\mathrm{E} \cdot \varphi(t, \mathrm{E}))>\lambda m^{2}
$$

is valid for a relatively dense set of values $\mathrm{t}$ on the axis $-\infty<\mathrm{t}<+\infty$ (at any $\lambda<1$ ).

Theorem IV is no longer valid, if the measure of the entire space $\mathrm{R}$ is infinite. Then it is already impossible to state that almost all motions are Poisson stable. At that, the point $p$ is called outgoing as $t \rightarrow+\infty$, if the trajectory $\varphi(t, p)$ has no $\omega$-limited points. Under these conditions, the Hopf theorem is valid.

Tеорема VII (Hopf). Let a locally compact metric space of motions with a countable base be given, wherein an invariant measure $\mu$ is defined with the following properties: $\mu \mathrm{R}=+\infty$ and for any compact set $\mathrm{F} \subset \mathrm{R}$ the measure $\mu \mathrm{F}$ is finite. Then almost all points $\mathrm{p} \in \mathrm{R}$ are either Poisson stable or outgoing as $\mathrm{t} \rightarrow+\infty$.

Khinchin's theorem shows that independently how many times a system visits any point in a bounded phase space R, it will revisit these points again and again.

For our purposes it is important to know how fast unstable points go to infinity? Let us consider a holonomic material system in an equilibrium position (when all the Lagrangian coordinates $\mathrm{q}_{1}, \mathrm{q}_{2}, \ldots, \mathrm{q}_{\mathrm{n}}$ are equal zero). For not too big deviations from the equilibrium, one can express the kinetic moment $\mathrm{T}$ and potential function $\mathrm{V}$ as quadratic forms

$$
T=\frac{1}{2} \sum_{i, j} a_{i j} \dot{q}_{i} \dot{q}_{j}, \quad V=\frac{1}{2} \sum_{i, j} b_{i j} q_{i} q_{j},
$$

where $a_{i j}=a_{j i}, b_{i j}=b_{j i}$. Substituting (66) into the equation of motion (the Lagrange equation of the second type [2]), we obtain:

$$
\sum_{i} a_{i j} \ddot{q}_{j}+\sum_{j} b_{i j} q_{j}=0 .
$$

At the transformation to the normal coordinates [17] the functions (66) are simplified:

$$
T=\frac{1}{2}\left(\dot{x}_{1}^{2}+\ldots+\dot{x}_{n}^{2}\right), \quad V=\frac{1}{2}\left(\lambda_{1} x_{1}^{2}+\ldots+\lambda_{n} x_{n}^{2}\right),
$$

where $\lambda_{1}, \ldots, \lambda_{n}$ are roots of the algebraic equation

$$
\Delta(\lambda) \equiv\left\|\lambda a_{i j}-b_{i j}\right\|=0 .
$$

At that, the Lagrange equations (67) are reduced to a number of $n$ independent equations

$$
\ddot{x}_{k}+\lambda_{k} x_{k}=0 . \quad(k=1, \ldots, n)
$$

According to Sylvester's theorem [17], all roots of the equation (68) are real. As a result, the solutions of (69) have the form: 


$$
\left.\begin{array}{ll}
\mathrm{x}_{\mathrm{k}}=\mathrm{A}_{\mathrm{k}} \cos \left(\mathrm{t} \sqrt{\lambda_{\mathrm{k}}}+\mathrm{B}_{\mathrm{k}}\right) & \text { for } \lambda_{\mathrm{k}}>0, \\
\mathrm{x}_{\mathrm{k}}=\mathrm{A}_{\mathrm{k}} \mathrm{t}+\mathrm{B}_{\mathrm{k}} & \text { for } \lambda_{\mathrm{k}}=0, \\
\mathrm{x}_{\mathrm{k}}=\mathrm{A}_{\mathrm{k}} \mathrm{e}^{\mathrm{t} \sqrt{-\lambda_{\mathrm{k}}}}+\mathrm{B}_{\mathrm{k}} \mathrm{e}^{-\mathrm{t} \sqrt{-\lambda_{\mathrm{k}}}} & \text { for } \lambda_{\mathrm{k}}<0,
\end{array}\right\}
$$

where $A_{k}, B_{k}$ are the integration constants defined by initial conditions.

From (70) one can see that only when all roots of the equation (68) are positive, the equilibrium is stable and its return leads to harmonic oscillations of normal coordinates $x_{k}$ with corresponding frequencies $\sqrt{\lambda_{k}}$. If only one root of the equation (68) is non-positive, then the state of equilibrium becomes unstable. At a slightest (infinitely small) perturbation the coordinate $x_{k}{ }^{*}$ corresponding to the non-positive root $\lambda_{k}{ }^{*}$ begins to increase with time under a linear (if $\lambda_{k}^{*}=0$ ) or an exponential law. Nevertheless, independently how far the normal coordinate $x_{k} *$ is from its initial position $x_{k}{ }^{*}=0$, at time inversion (i.e. at the replacement of $t$ by $-t$ and of all velocities $\dot{x}_{k}$ by $-\dot{x}_{k}$ ) all coordinates inevitably return to the initial position $x_{k}=0$ at $t=$ 0. (I. Loschmidt was the first who proposed such operation for a gas in 1876 [19] to refute Boltzmann's theory).

The example of stability/instability of normal oscillations made above at first seems to be too specific. However, it has all typical features of stability, instability, and determinism in the motion of mechanical systems (47). Moreover, it is proved in a general case [16] that the problem of stability of any solution of DE can be reduced to the problem of stability of the zero solution. In the vicinity of the equilibrium point (if it exists) it is always possible to reject the higher expansion terms of $\mathrm{T}$ and $\mathrm{V}$ in the right part of the equation (49) and thus to get their representation as quadratic forms (66).

If all roots of the equation (68) are positive, then the normal coordinates oscillate near the equilibrium position according to the expression in the upper line of the equation (70). At that, the oscillation amplitude is constant and is defined by the value of the first deviation $A_{k}$ caused by a momentary perturbation. Such motion is Lyapounov stable and the amplitudes $A_{k}$ define the number $\mathrm{h}$ in equation (62). In general case, a stable (Lyapounov stable) motion can be represented by a curve, in a ( $\mathrm{n}+$ 1)-dimensional space of the variables $\mathrm{q}_{1}, \ldots, \mathrm{q}_{\mathrm{n}}, \mathrm{t}$, surrounded by a tube [11], which possesses the property that every motion once appeared inside this tube remains inside it arbitrary long.

The motion of a set of non-interactive particles is completely unstable. In this case, all $\lambda_{\mathrm{k}}=0$ and, if each normal coordinate gets a momentary perturbation proportional to $A_{k}$, then all coordinates keep moving away from the initial position of rest in accordance with the expression in the second line of (70), i.e. under a linear law. Finally, if some roots of the equation (68) prove to be negative, then any (even infinitely small) momentary perturbation leads to the non-zero values of $A_{k}$ and $B_{k}$ with the same value $\mathrm{k}$ and then this normal coordinate $\mathrm{x}_{\mathrm{k}}$ deviates from the initial position under an exponential law (see the third line of (70)).

Let us note once more that in all cases discussed above, the trajectory after the time inversion operation turns back and absolutely deterministically goes along the way passed by it earlier until it returns to the initial point left by it at $\mathrm{t}=0$. But are the stochastization and loss of determinism possible in a classical dynamical system?

Fig. 1 shows that at elastic collisions of a light particle with motionless (i.e. very heavy) absolutely elastic and impenetrable sphere-like "atoms", the motion of the light particle after dozen and hundred of collisions acquires the properties of the Brownian movement (diffusion). It is because the probability of finding a light particle after time $t$ on a distance $r$ from the initial point $\mathrm{q}_{0}$ increases under the diffusion law:

$$
r \cong \operatorname{const} \sqrt{t} \cong \operatorname{const} \sqrt{N},
$$

where $\mathrm{N}$ is the number of collisions. No matter how far the light particle went from the zero point $\mathrm{q}_{0}$ as a result of $\mathrm{N}$ collisions, after the time inversion operation and $\mathrm{N}$ collisions in its backward motion the light particle will return to the initial point $\mathrm{q}_{0}$.

Fig. 2 shows that in a repetitive test when any small perturbation of initial trajectory occurs (by a small angle $\alpha_{0}$ ), already after two collisions the light particle changes its way qualitatively and will not go along the previous trajectory. However, there is no doubt that in the repetitive test the time inversion operation after $\mathrm{N}$ collisions will return the light particle back to the point $\mathrm{q}_{0}$ as a result of $\mathrm{N}$ backward collisions. Therefore, in classical dynamics (deterministic in principle), the problem of the nature of the perturbation $\alpha_{0}$ is still very important.

The determinism of classical motion will not change, if instead of impenetrable balls one will consider, for example, point-like particles with the Coulomb repulsive potential. If the repulsive Coulomb centers will reproduce the trajectory of Fig. 2, then the "diffusion" regularity (71) appears as well. However, at arbitrary large $\mathrm{t}$ (or N), the time inversion operation makes the light particle move backward along the trajectory until it returns exactly to the initial point $\mathrm{q}_{0}$ after $\mathrm{N}$ backward collisions. So, the "loss of memory" does not take place, in spite of the prediction in [19], if only there are no non-constructive operations of going to infinity, passages to the thermodynamic limit, etc.

G. A. Martynov [5] proved the variability of the global dynamical entropy, but not the fact that it only increases (see the 
equation (11)), so his optimism about the elimination of the contradiction between Newton's classical mechanics and the thermodynamics will hardly come true. All the more so that the Poincaré-Carathéodory recurrence theorems and particularly the Khinchin and Hopf theorems show that THERE ARE NO PERSPECTIVES TO REMOVE THIS CONTRADICTION. Also, one should not forget the opinion of high-experienced theorists L. D. Landau and E. M. Lifschitz on the impossibility to obtain the law of entropy increasing from the standard classical mechanics [8]. Then a seditious question arises: is everything correct with Newton's classical dynamics? And, in general, is this really mechanics, all this great mass of knowledge accumulated during more than 300 years and called now analytical (theoretical) classical mechanics?

\section{Realistic Mechanics. Elimination of the Poincaré and Zermelo Paradoxes}

Let us recall that W. Thomson and G. Helmholtz had worked on a theoretical model of vortex atoms since 1869 and W. Rankin was elaborating it as early as in 1850. By the end of the XIX century it was experimentally proved that atoms, molecules, and all matter in the Universe is composed of charged particles. At that, as early as in 1839 S. Earnshaw proved the theorem that a system of charges cannot be static and described by deterministic parameters. Thus, even well before the discovery of quantum mechanics and without any connection with it, L. Boltzmann and all his opponents beginning with A. Poincaré, should had come to a firm conclusion that atoms, molecules, and all other forms of matter in the Universe are not deterministic objects, but should be characterized by probabilistic parameters (mean values, variances, covariances, etc.). As for macro-bodies, they are all the more not deterministic, because the stars evolve, pulsate, explode, and on the planets there occur mountain formation, degassing, creation of a gas blanket or a liquid shell. So, even if not to take into account the accretion processes, the extraterrestrial objects cannot be simulated by "frozen" structure-free infinitely small points or structure-free absolutely elastic indestructible billiard balls. All mechanical objects are stochastic objects characterized by the expected values of their center of gravity or of their diameter and by an infinite (countable) set of momenta. Therefore the modeling of mechanical objects by infinitely small points (by the non-constructive Dirac delta-function), or by absolutely elastic billiard balls (by Heaviside's $\Theta$-function, whose derivative is the same non-constructive Dirac delta-function) is acceptable in the abstract science of mathematics, but not in mechanics dealing with real mechanical (physical) bodies. Strictly speaking, all that has been achieved in the last 300 years by Newton, Lagrange, and thousands of their followers, is not mechanics, but an abstract mechanical mathematics (AMM), i.e. a field of abstract mathematics, whose problems are induced by mechanics. The true real mathematical mechanics (RMM) should consider structured mechanical bodies. The structure should be characterized at least by a set of probabilistic parameters: by the expected value of the center of mass (of the diameter) and by a set of variances, covariances, etc.

From that it follows that the mathematical formalism of $R M M$ should be radically different from the $A M M$ formalism. But, may be, it would be sufficient to introduce a nonzero variance to the initial conditions of Cauchy's problem for Newton's equations (47) thus taking into account the structure of mechanical objects? However, that will not solve the problem. To see it, let us consider the T. Nishigory theorem [20].

Let us confine ourselves to the simplest case of a two-dimensional vector space $S$ formed by vectors $\mathrm{X}(\mathrm{t})$ and $\mathrm{Y}(\mathrm{t})$ with the inner product $(\mathrm{X}, \mathrm{Y})$. Let these vectors evolve with time according to the equations

$$
\begin{aligned}
& \frac{d X(t)}{d t}=a X(t)+b Y(t), \\
& \frac{d Y(t)}{d t}=d X(t)+c Y(t)
\end{aligned}
$$

with the initial conditions $\mathrm{X}(0)=\mathrm{X}, \mathrm{Y}(0)=\mathrm{Y}$. The vectors $\mathrm{X}$ and $\mathrm{Y}^{*}$ form an orthogonal basis in $S$, i.e. $\left(\mathrm{Y}^{*}, \mathrm{X}\right)=0$, where $\mathrm{Y}^{*}$ is found by the standard Schmidt orthogonalization procedure. We introduce a new vector

$$
Y^{*}(t)=Y(t)-w X(t) \text {, where } w=(\mathrm{Y}, \mathrm{X})(\mathrm{X}, \mathrm{X})^{-1},
$$

so that $\mathrm{Y}^{*}(0)=\mathrm{Y}^{*}$. Then the equations (72) transform into the following:

$$
\begin{aligned}
& \frac{d X(t)}{d t}=\alpha X(t)+b Y^{*}(t), \\
& \frac{d Y^{*}(t)}{d t}=\beta X(t)+\gamma Y^{*}(t),
\end{aligned}
$$

where $\alpha=a+b w, \beta=d+c w-w \alpha, \gamma=c-w b$. Excluding the variable $\mathrm{Y}^{*}(\mathrm{t})$ from (73), we obtain: 


$$
\frac{d X(t)}{d t}=\alpha X(t)+b \int_{0}^{t} N\left(t-t^{\prime}\right) \beta X\left(t^{\prime}\right) d t^{\prime}+b F(t),
$$

where functions $N(\mathrm{t})=\exp (\gamma t)$ and $F(\mathrm{t})=N(t) \mathrm{Y}^{*}$ satisfy the relations

$$
\begin{aligned}
& (F(t), X(0))=0, \\
& (F(t), F(0))=N(t)(F(0), F(0)) .
\end{aligned}
$$

When the initial conditions are fixed, we have a deterministic function $\mathrm{F}(\mathrm{t})$. If the initial variables $\mathrm{Y}$ and/or $\mathrm{X}$ have a statistical distribution, then $\mathrm{Y}^{*}$ and $\mathrm{F}(\mathrm{t})$ become stochastic variables, and the equation (74) becomes a non-Markovian Langevin equation, where the upper and the lower relation (75) express the causality condition and the fluctuation-dissipation theorem, respectively. At that the scalar product is averaged over the initial distribution.

It follows from the Nishigory theorem that if a deterministic initial distribution is represented by the Dirac delta-function, then the time evolution of the system is described by a deterministic solution to a Cauchy problem for the corresponding DE. However, as soon as the smallest uncertainty (stochasticity) appears in the initial conditions, the formalism of the abstract classical Newtonian mechanical mathematics (Cauchy's problem for the equations (47)) should be substituted by a stochastic formalism of the Langevin equation type. But for stochastic processes the theorems of Poincaré, Hopf, and Khinchin are not valid at all! Instead, the ergodic theorems are valid for them [21,22]. Theorems and deductions of the RMM are essentially different from those of AMM. Only in rare cases, for stable motions with a constant cross-section of the tube around a stable trajectory, the solution of the initial problem for stochastic DE can be factorized into a product of the distribution function of the RMM initial conditions and the stable trajectory obtained in the AMM. Fig. 3 shows a picture of real motion described by the RMM that generalizes an abstract (mathematical) motion in the framework of the AMM and corresponds to the equation (61) for the case of a stable motion in a tube of constant cross-section $2 \sigma$. If at the initial time $t_{0}$ the probability density is represented by the normal distribution [21]

$$
P\left(y, t_{0}\right)=\frac{1}{\sqrt{2 \pi} \sigma} \exp \left[-\frac{\left(y-y_{0}\right)^{2}}{2 \sigma^{2}}\right],
$$

then, for the situation shown in Fig. 3, one can immediately get $P(y, t)$ at any subsequent time $t$ :

$$
P(y, t)=\frac{1}{\sqrt{2 \pi} \sigma} \exp \left[-\frac{(y-y(t))^{2}}{2 \sigma^{2}}\right] .
$$

Here $y(t)$ is an explicit function of time obtained in the framework of the AMM as a result of a solution of Cauchy's problem for the deterministic equation (61). In particular, it may be the upper line of (70).

Let us now proceed to a simplest unstable motion in the context of the RMM, which corresponds to the second line in the equation (70). Then the picture of motion will be shown by the left part of Fig. 4. Here it is still possible to construct the function $P(\mathrm{y}, t)$, if a "mathematical" trajectory $\mathrm{y}(t)$ is known. It is shown in the left part of Fig. 4 that the normal distribution $P(\mathrm{y}, t)$ is conserved on the length of a "spreading motion" of $N$ particles from $t_{0}$ to $t_{2}$. However, unlike the situation on Fig. 3, the variance on Fig. 4 increases linearly with time (from $\sigma_{0}$ at $t_{0}$ up to $2 \sigma_{0}$ at $t_{1}$ ). The situation changes drastically after a first collision at $t_{2}$ (it is shown in the right part of Fig. 4 attached eclectically to the left part). After a first collision, the probability density $P(\mathrm{y}, t)$ does not only loose the symmetry of (77), but acquires discontinuities and other singularities.

Now it is clear that from all what has been made for 300 years of a "victorious march of $N$ body paradigm" [11], only results obtained for stable "mathematical" motions are applicable to the physical mechanics of real (not abstract structure-free bodies). That means that the Poincaré recurrence theorem, as well as the Zermelo and Loschmidt paradoxes are valid only in the framework of the AMM, i.e. for structure-free mathematical objects. As for real (structured) mechanical objects/bodies, Poincaré's theorem and Zermelo's paradox are applicable only to the class of stable motions with a constant tube. For unstable motions of real bodies, the theorems of Picard, Poincaré, Khinchin, etc. should be replaced by limiting and ergodic theorems of the theory of stochastic processes, that means that the Laplacian (mechanical) determinism does not exist in the RMM. For example, according to the Khinchin theorem, the trajectory of a structure-free mathematical point again and again revisits almost any point of a bounded phase space. On the contrary, any configuration in the RMM, if not limiting, repeats itself only with the probability of measure zero. In other words, unlike in the AMM, the following ergodic replacement of the Khinchin theorem is applicable to the RMM:

If a motion is unstable, then the bunch of trajectories starting from the initial set $\mathrm{E}$ at $t=t_{0}$, can be collected in $\mathrm{E}$, at any $t>t_{0}$, only with the probability zero, if only the set $\mathrm{E}$ is not ergodic (i.e. that can be filled with unstable trajectories as $t \rightarrow \infty$ ).

In the RMM, the Liouville theorem should be also replaced. In order to find a substitute let us make a small excursus in the theory of stochastic differential equations (SDE) [23]. In the theory of the Brownian motion, well known is the Langevin 
equation:

$$
m \frac{d^{2} \vec{r}}{d t^{2}}+h \frac{d \vec{r}}{d t}+\operatorname{gradU}(\vec{r}, t)=\vec{F}(t),
$$

where $\mathrm{m}$ is the particle mass, $\mathrm{U}$ is the external potential, $\mathrm{h}(\mathrm{dr} / \mathrm{dt})$ is the friction force, $\mathrm{F}(\mathrm{t})$ is a normally distributed (Gaussian) random force with the correlation function

$$
\left\langle F_{i}\left(t_{1}\right) F_{j}\left(t_{2}\right)\right\rangle=B_{i j}\left(t_{1}-t_{2}\right)=2 B \delta_{i j} \delta\left(t_{1}-t_{2}\right)=2 k T h \delta_{i j} \delta\left(t_{1}-t_{2}\right) .
$$

The Langevin equation is a very special case of the general Ito's SDE for stochastic processes $\xi(\mathrm{t})$ :

$$
d \xi(t)=a(t, \xi(t)) d t+\sqrt{b(t, \xi(t))} d w(t),
$$

where $a$ and $b$ are ordinary (deterministic) functions, $w(t)$ is a stochastic Wiener process, i.e., a uniform Gaussian process with independent increments. For this process

$$
w(0)=0, \quad\langle w(t)\rangle=0, \quad \operatorname{Pr} o b .\{a<w(t)<b\}=\frac{1}{\sqrt{2 \pi t}} \int_{a}^{b} e^{-\frac{z^{2}}{2 t}} d z .
$$

Under certain conditions (if functions $a(t, x)$ and $\sqrt{b(t, x)}$ satisfy the conditions of the uniqueness theorem for SDE), the SDE solutions (79) prove to be equivalent [23] to the solutions of the following reverse Kolmogorov diffusion equation:

$$
\frac{\partial u(t, x)}{\partial t}+a(t, x) \frac{\partial u(t, x)}{\partial x}+\frac{1}{2} \sigma^{2}(t, x) \frac{\partial^{2} u(t, x)}{\partial x^{2}}=0,
$$

where $\sigma(t, x)=\sqrt{b(t, x)}$, and the function $u$ is the expected value of an arbitrary twice continuously differentiable function $f(x)$ :

$$
u(t, x)=\left\langle f\left(\xi_{x, t}(s)\right)\right\rangle, 0 \leq t \leq s, \quad x \in(-\infty, \infty) .
$$

I. I. Gikhman and A. V. Skorokhod [23] have shown that Ito's equation (79) is far from exhausting all the types of stochastic processes. That is, in a very general case of processes without aftereffect the stochastic process $\xi(\mathrm{t})$ should obey the Ito-Gikhman-Skorokhod SDE:

$$
d \xi(t)=a(t, \xi(t)) d t+b(t, \xi(t)) d w(t)+\int c(t, \xi(t), z) \bar{v}(d t, d z)
$$

where $a, b$ and $c$ are ordinary (deterministic) functions, $\bar{v}_{t x}(h, A)$ is the Poisson probability measure:

$$
\begin{aligned}
& \bar{v}_{t x}(h, A)=v_{t x}(h, A)-h \Pi_{t x}(A), \\
& h \Pi_{t x}(A)=\left\langle v_{t x}(h, A)\right\rangle,
\end{aligned}
$$

$v(h, A)$ is the standard Poisson measure $t \Pi(A)=\langle v(t, A)\rangle=D^{2}\{v\}$, generated by the Poisson distribution

$$
\mathrm{P}_{x}(t)=\frac{(\lambda t)^{x}}{x !} e^{-\lambda t}
$$

A continuous stochastic Ito process (79) can be obtained from a more general process (82), but only if the jump function $\mathrm{c}(\mathrm{t}$, $\mathrm{x}, \mathrm{z})$ equals zero.

If SDE (82) satisfies the conditions of existence and uniqueness of the solution and functions $a, b$, and c together with their gradients are square integrable, then the following reverse Kolmogorov-Gikhman-Skorokhod equation [23] is valid for the function $\mathrm{u}(\mathrm{t}, \mathrm{x})$ : 


$$
\begin{aligned}
& \frac{\partial u(t, x)}{\partial t}-\pi u(t, x)+(a(t, x)-\lambda(t, x)) \nabla u(t, x)+\frac{1}{2} s p\left(\nabla^{2} u(t, x) b(t, x) b *(t, x)\right)+ \\
& +\int u(t, x+c(t, x, z)) \Pi(d z)=0
\end{aligned}
$$

where the intensity of jumps is $\pi=\int \Pi(d z)$, and their mean amplitude is

$$
\lambda(x)=\int c(x, z) \Pi(d z)
$$

Also, the forward Kolmogorov-Gikhman-Skorokhod equation has been obtained:

$$
\frac{\partial P(t, x ; \tau, y)}{\partial \tau}+\sum_{i=1}^{n} \frac{\partial\left(\bar{a}_{i} P\right)}{\partial y_{i}}=\frac{1}{2} \sum_{i, j=1}^{n} \frac{\partial^{2}\left(\sigma_{i j}(\tau, y) P\right)}{\partial y_{i} \partial y_{j}}+\pi P+\int P\left(t, x ; \tau, y-c^{*}(t, y, z)\right) D(y) \Pi(d z),
$$

where $\bar{a}_{i}=\bar{a}_{i}(t, x)=a_{i}(t, x)-\lambda_{i}(t, x)$, and $D(y)$ is the functional determinant of the inverse mapping $y=u-c^{*}(\tau, u, z)$. The second term in the left part of this SDE is the deterministic "drift", the first term in the right part is the diffusion term corresponding to continuous infinitesimal jumps, while the second and the third terms in it represent the contribution of discrete jumps of finite amplitude.

Now, instead of the deterministic Newton 2-nd law of the AMM one can write down a stochastic equation of motion of the RMM for a system of N particles described by the Hamilton function (12) [24] :

$$
\frac{\partial F_{N}}{\partial t}+\sum_{i=1}^{N}\left\{\frac{\partial F_{N}}{\partial \vec{r}_{i}} \frac{\partial H}{\partial \vec{p}_{i}}-\frac{\partial F_{N}}{\partial \vec{p}_{i}} \frac{\partial H}{\partial \vec{r}_{i}}\right\}=\sum_{i=1}^{N}\left\{\frac{1}{2}\left(\frac{d \sigma_{r_{i}}^{2}\left(\Delta \vec{r}_{i}, v_{i} t\right)}{d t}\right) \frac{\partial^{2} F_{N}}{\partial \vec{r}_{i}^{2}}+\frac{1}{2}\left(\frac{d \sigma_{p_{i}}^{2}\left(\Delta \vec{p}_{i}, g_{i} t\right)}{d t}\right) \frac{\partial^{2} F_{N}}{\partial \vec{p}_{i}^{2}}\right\}
$$

where $F_{N}$ is the $N$-particle distribution function (13) normalized to 1 ;

$$
\sigma_{r_{i}}^{2} \text { and } \sigma_{p_{i}}^{2}
$$

are variances of coordinates and momentum of the $i$-type particle, respectively, $v_{i}$ is the velocity of the $i$-th particle, $g_{i}$ is its acceleration. In order to obtain the time evolution of the system, a Cauchy problem should be solved for the DE (85) with an initial condition at $t=t_{0}$ in, for instance, a Gaussian form:

$$
F_{N}\left(t_{0}, \vec{r}_{1}, \ldots, \overrightarrow{p_{N}}\right)=\prod_{i=1}^{N} \frac{1}{\Delta \overrightarrow{r_{i}} \sqrt{2 \pi}} e^{-\frac{\left(\overrightarrow{r_{i}}-\overrightarrow{r_{i 0}}\right)^{2}}{2\left(\Delta \vec{r}_{i}\right)^{2}}} \frac{1}{\Delta \overrightarrow{p_{i}} \sqrt{2 \pi}} e^{-\frac{\left(\overrightarrow{p_{i}}-\overrightarrow{p_{i 0}}\right)^{2}}{2\left(\Delta \overline{p_{i}}\right)^{2}}}
$$

If all variances (86) in the equation (85) are constant (that takes place in the case of stable motion), then their time derivatives equal zero, the right part of the equation of motion (85) vanishes and the equation (85) degenerates into the Liouville equation (14). The latter immediately implies the Liouville theorem on the conservation of the phase space flow, Poincaré's and Loschmidt's paradoxes, BBGKY and Martynov hierarchy equations, constancy of the global entropy, and the incompatibility with the Boltzmann kinetic equation and his $\mathrm{H}$-theorem.

Such mathematical situation corresponds to the upper line in the equation (70), but really it is not attained exactly even on planet orbits. As a result, the "planet gas" around the Sun gradually "evaporates": one can expect in the nearest 2-4 billions of years that one of the planets or big planetoids (perhaps, Pluto or Sedna) will leave the Solar system.

However, if even one of the variances in (86) has a nonzero derivative, the equation (85) retains its diffusional nature, which immediately implies ergodic theorems for stochastic processes, including the law of entropy increasing [25]. Thus, it is just an abstract mathematical theory, the Newtonian mechanical mathematics, is incompatible with the kinetic Boltzmann equation and his $\mathrm{H}$-theorem. On the contrary, the realistic mathematical mechanics immediately implies the law of entropy increasing even for the systems of two particles without a passage to the thermodynamic limit or other non-constructive manipulations with infinities.

It should be noted that our result is more "liberal" as compared with the Nishigori theorem. The latter states that the deterministic Newton mechanics is not valid even at a slightest uncertainty of initial data. And our equation (85) shows that the deterministic AMM becomes invalid only when at least one variance is nonzero. However, there is no contradiction here. The factorization (77) takes place just at a nonzero but fixed variance (that corresponds to a steady motion in a tube of a constant cross-section), when the stochastic technique can be already applied, but the Newtonian AMM still has not lost its validity.

Let us present solutions of Cauchy's problem for the DE (85) with the initial condition (87) for two simplest cases. A first 
case is that of a stable one-dimensional motion of a particle of mass $m$ and acceleration $\mathrm{g}$. The stability of motion means that the variances (86) of coordinate $\left(\sigma_{x}^{2}=(\Delta x)^{2}\right)$ and momentum $\left(\sigma_{p}^{2}=(\Delta p)^{2}\right)$ are constant. Then the derivatives $\frac{d}{d t} \sigma_{x}^{2}$ and $\frac{d}{d t} \sigma_{p}^{2}$ are equal to zero and the DE (85) becomes simpler:

$$
\frac{\partial F(t, x, p)}{\partial t}+\frac{p}{m} \frac{\partial F(t, x, p)}{\partial x}+g \frac{\partial F(t, x, p)}{\partial p}=0
$$

The initial condition (87) becomes

$$
F(0, x, p)=\frac{1}{\Delta x \sqrt{2 \pi}} e^{-\frac{\left(x-x_{0}\right)^{2}}{2(\Delta x)^{2}}} \frac{1}{\Delta p \sqrt{2 \pi}} e^{-\frac{\left(p-p_{0}\right)^{2}}{2(\Delta p)^{2}}} .
$$

By a standard technique [26] one gets the solution of Cauchy's problem for the DE (88) with the initial condition (89) and the boundary condition $F(t, x, p) \rightarrow 0$ as $|\mathrm{x}||\mathrm{p}| \rightarrow \infty$ :

$$
F(t, x, p)=\frac{1}{2 \pi \sigma_{0 x} \sigma_{0 p}} \exp \left(-\frac{\left(x-\bar{x}(t)-\frac{(p-\bar{p}(t))}{m} t\right)^{2}}{2 \sigma_{0 x}^{2}}-\frac{(p-\bar{p}(t))^{2}}{2 \sigma_{0 p}^{2}}\right)
$$

where

$$
\bar{x}(t)=\overline{x_{0}}+\frac{\overline{p_{0}}}{m} t+\frac{g}{2 m} t^{2} \bar{p}(t)=\bar{p}_{0}+g t
$$

Let us calculate the Shennon entropy for the packet (90):

$$
S=-\int_{-\infty}^{\infty} \int_{-\infty}^{\infty} F(t, x, p) \ln F(t, x, p) d x d p=1+\ln \left(2 \pi \sigma_{0 x} \sigma_{0 p}\right) .
$$

The expressions (90) and (91) are time inverse invariant. That is in general true for any distribution function $\mathrm{F}(\mathrm{t}, \mathrm{x}, \mathrm{p})$ satisfying the Liouville equation, as according to the Liouville theorem, the total derivative $(\mathrm{dF} / \mathrm{dt})$ of the $\mathrm{F}(\mathrm{t}, \mathrm{x}, \mathrm{p})$ is always zero:

$$
\frac{d}{d t} S(t)=-\frac{d}{d t} \int F(t, x, p) \ln F(t, x, p) d x d p=-\int(1+\ln F) \frac{d F}{d t} d x d p=0,
$$

A second case is that of a one-dimensional uniform unstable motion of one particle of mass $m$ and acceleration $g$. Here the variances of momentum and coordinate increase with time.

The equation of motion (85) takes the form

$$
\frac{\partial F(t, x, p)}{\partial t}+\frac{p}{m} \frac{\partial F(t, x, p)}{\partial x}+g \frac{\partial F(t, x, p)}{\partial p}=\frac{1}{2} \kappa g^{2} \cdot \frac{\partial^{2} F(t, x, p)}{\partial p^{2}} .
$$

The integration of (93) with the initial conditions (89) and the boundary condition $F(t, x, p) \rightarrow 0$ as $|\mathrm{x}||\mathrm{p}| \rightarrow \infty$ gives:

$$
\mathrm{F}(\mathrm{t}, \mathrm{x}, \mathrm{p})=\frac{1}{2 \pi \sqrt{\mathrm{R}(\mathrm{t})}} \exp \left(-\frac{\mathrm{Q}(\mathrm{t}, \mathrm{x}, \mathrm{p})}{2 \mathrm{R}(\mathrm{t})}\right),
$$

where

$$
R(t)=\sigma_{x}^{2}(t) \sigma_{p}^{2}(t)-\sigma_{x p}^{2}(t)
$$




$$
\begin{gathered}
Q(t, x, p)=\sigma_{p}^{2}(t)(x-\bar{x}(t))^{2}+\sigma_{x}^{2}(t)(p-\bar{p}(t))^{2}-2 \sigma_{x p}(t)(x-\bar{x}(t))(p-\bar{p}(t)), \\
\sigma_{x}^{2}(t)=\sigma_{0 x}^{2}+\frac{\sigma_{p}^{2}(t)}{m^{2}} t^{2}+\frac{1}{3} \frac{g^{2}}{m^{2}} \kappa t^{3} \\
\sigma_{p}^{2}(t)=\sigma_{0 p}^{2}+g^{2} \kappa t \\
\sigma_{x p}(t)=\frac{\sigma_{0 p}^{2}}{m} t+\frac{g^{2}}{2 m} \kappa \cdot t^{2}
\end{gathered}
$$

The Shannon entropy for distribution functions (94) is

$$
S(t)=-\int_{-\infty}^{\infty} \int_{-\infty}^{\infty} F(t, x, p) \ln F(t, x, p) d x d p=1+\ln \left(2 \pi \sigma_{x}(t) \sigma_{p}(t) \chi_{x p}(t)\right),
$$

where $\chi_{x p}(t)=\sqrt{1-\rho_{x p}^{2}(t)}$, and $\rho_{x p}(t)=\sigma_{x p}(t) / \sigma_{x} \sigma_{p}$ is the coefficient of the correlation of $x$ with $p$. At the inversion of time the entropy (96) becomes imaginary, therefore the evolution of the probability packet (94) is time irreversible.

The results obtained allow one to critically review the above mentioned theorem of Nishigory [20]. From (90), (91) one can see that the Hamilton mechanics predicts a determined and reversible evolution of a mechanical system for the initial conditions in the form of the Gaussian distribution. It follows from (95), (96) that the equation (93) is a stochastic Langevin equation not because of a statistical character of initial data for the equation (88), but only due to stochastic components of interparticle interaction, i.e. due to a passage from the classical to the complete Liouville equation.

It is impossible to obtain the irreversibility in time by a choice of initial conditions in Newton's equations (or in the corresponding Liouville equation) for any mechanical system of point-like structureless bodies with nonzero mass. One needs to introduce into the potentials of interparticle interaction, stochastic corrections determined by (dynamical) structure of real, not point-like bodies.

\section{Evolution of a Probability Packet in the Deterministic Relativistic Mechanics}

The evolution of a probability packet for a free relativistic particle was studied earlier in [31], [32]. We consider a more general case of a relativistic particle uniformly accelerated by the action of another body of much greater mass. In the relativistic case the Liouville equation for a system of $N$ particles should be written in a covariant form:

$$
\sum_{i=1}^{N} p_{i}^{\mu} \partial_{\mu} F_{N}+\sum_{i=1}^{N} m_{i}^{2} g_{i}^{\mu} \frac{\partial}{\partial p_{i}^{\mu}} F_{N}=0
$$

where $F_{N}$ is a $N$-particle distribution function of 4-coordinats $x_{i}^{\mu}$ and 4-momenta $p_{i}^{\mu}$, normalized to $1, g_{i}^{\mu}$ are the 4-accelerations.

Suppose that the initial dimensionless distribution normalized to 1 has a Gaussian form in each variable.

$$
F_{N}\left(x_{1}^{\mu}, \ldots, x_{N}^{\mu}, p_{1}^{\mu}, \ldots, p_{N}^{\mu}\right)=\prod_{i=1}^{N} \frac{1}{\sqrt{2 \pi} \sigma_{r 0}} e^{-\frac{\left(x_{i}-\hat{x}_{00}\right)_{\mu}\left(x_{i}-x_{i 0}\right)^{\mu}}{2 \sigma_{00}^{2}}} \frac{1}{\sqrt{2 \pi} \sigma_{p 0}} e^{-\frac{\left(p_{i}-p_{i 0}\right)_{\mu}\left(p_{i}-p_{i 0}\right)^{\mu}}{2 \sigma_{p 0}^{2}}}
$$

where $x_{\text {i } 0}^{\mu}, p_{\text {io }}^{\mu}, \sigma_{r 0}^{2}=\sigma_{\mu r_{0 i}} \sigma_{r_{0 i}}^{\mu}$, and $\sigma_{p 0}^{2}=\sigma_{\mu p_{0 i}} \sigma_{p_{0 i}}^{\mu}$ are the initial average values and variances of the 4-coordinate and 4-momentum of the i-th particle, respectively.

The solution of the equation (97) with the initial conditions (98) and the boundary conditions

$F_{N}\left(x_{1}^{\mu}, \ldots, x_{N}^{\mu}, p_{1}^{\mu}, \ldots, p_{N}^{\mu}\right) \rightarrow 0$ as $x_{\mathrm{i}}, p_{\mathrm{i}} \rightarrow \propto$ has the form:

$$
F_{N}\left(x_{1}^{\mu}, \ldots, x_{N}^{\mu}, p_{1}^{\mu}, \ldots, p_{N}^{\mu}\right)=\prod_{i=1}^{N} \frac{1}{\sqrt{2 \pi \sigma_{\mu r_{i}} \sigma_{r_{i}}^{\mu}}} e^{-\frac{\left(x_{i}-\bar{x}_{i}\right)_{\mu}\left(x_{i}-\bar{x}_{i}\right)^{\mu}}{2 \sigma_{\mu r_{i}} \sigma_{r i}^{\mu}}} \frac{1}{\sqrt{2 \pi \sigma_{\mu p_{i}} \sigma_{p_{i}}^{\mu}}} e^{-\frac{\left(p_{i}-\bar{p}_{i}\right)_{\mu}\left(p_{i}-\bar{p}_{i}\right)^{\mu}}{2 \sigma_{\mu p_{i}} \sigma_{p_{i}}^{\mu}}}
$$


where $\bar{x}_{i}^{\mu}, \bar{p}_{i}^{\mu}$ are the average values of 4-coordinates and 4-momenta.

The integration in a 4-coordinate or a 4-momentum gives a distribution only in the 4-coordinate or the 4-momentum subspace of the phase space $\left(x_{i}^{\mu}, p_{i}^{\mu}\right)$

$$
\begin{aligned}
& F_{N}\left(x_{1}^{\mu}, \ldots, x_{N}^{\mu}\right)=\prod_{i=1}^{N} \frac{1}{\sqrt{2 \pi \sigma_{\mu r_{i}} \sigma_{r_{i}}^{\mu}}} e^{-\frac{\left(x_{i}-\bar{x}_{i}\right)_{\mu}\left(x_{i}-\bar{x}_{i}\right)^{\mu}}{2 \sigma_{\mu r_{i}} \sigma_{r_{i}}^{\mu}}} \\
& F_{N}\left(p_{1}^{\mu}, \ldots, p_{N}^{\mu}\right)=\prod_{i=1}^{N} \frac{1}{\sqrt{2 \pi \sigma_{\mu p_{i}} \sigma_{p_{i}}^{\mu}}} e^{-\frac{\left(p_{i}-\bar{p}_{i}\right)_{\mu}\left(p_{i}-\bar{p}_{i}\right)^{\mu}}{2 \sigma_{\mu \mu p_{i}} \sigma_{p_{i}}^{\mu}}}
\end{aligned}
$$

In the case of a uniform unstable motion of a particle of mass $m$ with the 4-velocity $u^{\mu}$

and the 4-acceleration $g^{\mu}=\frac{1}{m c} \frac{d p^{\mu}}{d s}$ the variance of the 4-momentum $\sigma_{p}^{2}=\sigma_{\mu p_{i}} \sigma_{p_{i}}^{\mu}=(\Delta p)^{2}$ is constant and $x=s_{0}-(p / m c) s-(g / 2) s^{2}$. The variance of 4-coordinate $\sigma_{x}^{2}=(\Delta x)^{2}$

grows as the interval changes. So, for this type of relativistic packet the variance $\sigma^{2}(t)$ grows as the interval changes:

$$
\sigma^{2}(s)=\sigma_{0}^{2}+(\Delta u)^{2} s^{2}
$$

where $\Delta u$ is the width of the wave packet. Thus, in this case $\sigma_{x}^{2}=(\Delta x)_{0}{ }^{2}+(\Delta u)^{2} s^{2}, \quad \sigma_{p}^{2}=(\Delta p)^{2}$.

Following [33] -[35], we introduce the 4- entropy $S^{v}$ for the one-particle distribution function:

$$
S^{v}=-\int \frac{d^{3} p}{p^{0}} F \ln F
$$

One can see that the Shannon entropy is the $S^{0}$ component of $S^{\nu}$ :

$$
S=S^{0}=-\int F \ln F d^{3} p
$$

Now we calculate the Shannon entropy of packets for a one-particle distribution function:

$$
\begin{gathered}
S=-\int_{-\infty}^{+\infty} F\left(x^{\mu}, p^{\mu}\right) \ln F\left(x^{\mu}, p^{\mu}\right) d x^{\mu} d p^{\mu}=1+\ln \left(2 \pi \sqrt{\sigma_{\mu r} \sigma_{r}^{\mu} \sigma_{\mu p} \sigma_{p}^{\mu}}\right) \\
S_{x}=-\int_{-\infty}^{+\infty} F\left(x^{\mu}\right) \ln F\left(x^{\mu}\right) d x^{\mu}=\frac{1}{2}+\ln \left(\sqrt{2 \pi \sigma_{\mu r} \sigma_{r}^{\mu}}\right) \\
S_{p}=-\int_{-\infty}^{+\infty} F\left(p^{\mu}\right) \ln F\left(p^{\mu}\right) d p^{\mu}=\frac{1}{2}+\ln \left(\sqrt{2 \pi \sigma_{\mu p} \sigma_{p}^{\mu}}\right)
\end{gathered}
$$

As the expressions (98) and (102) are relativistic invariants, they should also be time- reversal invariants. As for the packet (100), its width and entropy grow with time according to (102). That is true if one substitutes $t$ by $-t$. Thus, like non-relativistic mechanics, the deterministic relativistic mechanics is reversible in time.

\section{The Spreading of a Probability Packet in the Stochastic Relativistic Mechanics}

In the relativistic case a simplest dynamical model of a system of material points with stochastic interaction may be described by a system of stochastic DE

$$
\frac{d x^{\mu}}{d s}=\frac{p^{\mu}}{m c},
$$




$$
\frac{d p^{\mu}}{d s}=m c g^{\mu}(1+\xi(s))
$$

where $\xi(s)$ is the Gaussian white noise of intensity $\kappa$ :

$$
\langle\xi(s)\rangle=0, \quad\langle\xi(s) \xi(s+\sigma)\rangle=\kappa \delta(\sigma)
$$

Here $\langle\ldots\rangle$ symbolizes an averaging over the probability density function.

Let us take the initial conditions

$$
\bar{x}_{0}^{\mu}, \bar{p}_{0}^{\mu}, \sigma_{r 0}^{2}=\sigma_{\mu r_{0}} \sigma_{r_{0}}^{\mu}, \sigma_{p 0}^{2}=\sigma_{\mu p_{0}} \sigma_{p_{0}}^{\mu} .
$$

The expected values and variances of the 4-coordinate and 4-momentum correspond to the initial interval. In the nonrelativistic case a stochastic generalization of the non-stationary Liouville equation for the probability density can written in a covariant form:

$$
p^{\mu} \partial_{\mu} F\left(x^{\mu}, p^{\mu}\right)+m^{2} g^{\mu} \frac{\partial}{\partial p^{\mu}} F\left(x^{\mu}, p^{\mu}\right)=\frac{1}{2} \kappa g_{\mu} g^{\mu} \frac{\partial}{\partial p^{\mu}} \frac{\partial}{\partial p_{\mu}} F\left(x^{\mu}, p^{\mu}\right)
$$

Without contribution of the stochastic force $\xi(s)$ the equation (107) transforms into the relativistic Liouville equation (97). The solution of the equation (107) with the initial conditions (98) and the boundary conditions $F\left(x^{\mu}, p^{\mu}\right) \rightarrow 0$ as $x^{\mu}, p^{\mu}$ $\rightarrow \propto$ gives the normalized probability density function

$$
F\left(x^{\mu}, p^{\mu}\right)=\frac{1}{2 \pi \sqrt{R(s)}} \exp \left(-\frac{Q\left(x^{\mu}, p^{\mu}\right)}{2 R(s)}\right)
$$

where

$$
\begin{gathered}
R(s)=\sigma_{x}^{2}(s) \sigma_{p}^{2}(s)-\sigma_{x p}^{2}(s), \\
Q\left(x^{\mu}, p^{\mu}\right)=\sigma_{p}^{2}(s)\left(x_{\mu}-\bar{x}_{\mu}\right)\left(x^{\mu}-\bar{x}^{\mu}\right)+\sigma_{x}^{2}(s)\left(p_{\mu}-\vec{p}_{\mu}\right)\left(p^{\mu}-\vec{p}^{\mu}\right)-2 \sigma_{x p}^{2}(s)\left(x_{\mu}-\bar{x}_{\mu}\right)\left(p^{\mu}-\vec{p}^{\mu}\right) . \\
\sigma_{x p}(s)=\left\langle\left(x_{\mu}-\vec{x}_{\mu}\right)\left(p^{\mu}-\vec{p}^{\mu}\right)\right\rangle .
\end{gathered}
$$

Integrating (108) in $x^{\mu}$ and $p^{\mu}$, we can obtain normalized probability functions in configuration and momentum subspaces respectively:

$$
\begin{gathered}
F\left(x^{\mu}\right)=\int_{-\infty}^{\infty} F\left(x^{\mu}, p^{\mu}\right) d p^{\mu}=\frac{1}{\sqrt{2 \pi} \sigma_{x}(s)} \exp \left(-\frac{\left(x_{\mu}-\vec{x}_{\mu}\right)\left(x^{\mu}-\vec{x}^{\mu}\right)}{2 \sigma_{x}^{2}(s)}\right), \\
F\left(p^{\mu}\right)=\int_{-\infty}^{\infty} F\left(x^{\mu}, p^{\mu}\right) d x^{\mu}=\frac{1}{\sqrt{2 \pi} \sigma_{p}(s)} \exp \left(-\frac{\left(p_{\mu}-\vec{p}_{\mu}\right)\left(p^{\mu}-\vec{p}^{\mu}\right)}{2 \sigma_{p}^{2}(s)}\right) .
\end{gathered}
$$

If one formally integrates (105), one gets

$$
p^{\mu}=\eta^{\mu}+m c g^{\mu} s+m c g^{\mu} \int_{s_{0}}^{s} \xi(\sigma) d \sigma .
$$

If the initial value of the stochastic process is $p^{\mu}$, then the Gaussian stochastic variable $\eta^{\mu}$ will satisfy the conditions

$$
\left\langle\eta^{\mu}\right\rangle=\vec{p}_{0}^{\mu}, \quad\left\langle\eta_{\mu} \eta^{\mu}\right\rangle=\sigma_{p 0}^{2}
$$

The process $p^{\mu}$ is Gaussian [36]. Let us calculate its first two moments: 


$$
\begin{aligned}
& \vec{p}^{\mu}=\left\langle p^{\mu}\right\rangle=\left\langle\eta^{\mu}+m c g^{\mu} s+m c g^{\mu} \int_{s_{0}}^{s} \xi(\sigma) d \sigma\right\rangle=\vec{p}_{0}^{\mu}+m c g^{\mu} s . \\
& \sigma_{p}^{2}=\left\langle p_{\mu} p^{\mu}\right\rangle-\vec{p}_{\mu} \vec{p}^{\mu}=\sigma_{p 0}^{2}+m^{2} c^{2} g_{\mu} g^{\mu}\left\langle\left(\int_{s_{0}}^{s} \xi(\sigma) d \sigma\right)^{2}\right\rangle= \\
& =\sigma_{p 0}^{2}+\kappa m^{2} c^{2} g_{\mu} g^{\mu} \int_{s_{0}}^{s} d \sigma \int_{s_{0}}^{s} \delta\left(\sigma-\sigma^{\prime}\right) d \sigma^{\prime}=\sigma_{p 0}^{2}+\kappa m^{2} c^{2} g_{\mu} g^{\mu} s .
\end{aligned}
$$

As a Gaussian process is completely characterized by its expected value and variance [36], the expressions (116), (117) determine a unique distribution function (108) in the subspace of 4-momenta.

Analogously, a formal integration of the equation (105) with the indicated above initial conditions gives

$$
x^{\mu}=\zeta^{\mu}+\frac{1}{m} \int_{s_{0}}^{s} p^{\mu}(\sigma) d \sigma=\zeta^{\mu}+\frac{1}{m} \int_{s_{0}}^{s}\left(\eta^{\mu}+m c g^{\mu} \sigma+m c g^{\mu} \int_{\sigma_{0}}^{\sigma} \xi\left(\sigma^{\prime}\right) d \sigma^{\prime}\right) d \sigma
$$

where $\zeta^{\mu}$ is a Gaussian stochastic variable:

$$
\left\langle\zeta^{\mu}\right\rangle=\vec{x}_{0}^{\mu}, \quad\left\langle\zeta_{\mu} \zeta^{\mu}\right\rangle=\sigma_{x 0}^{2}
$$

Hence

$$
\begin{gathered}
\vec{x}^{\mu}=\left\langle x^{\mu}\right\rangle=\left\langle\zeta^{\mu}+\frac{1}{m} \int_{s_{0}}^{s} p^{\mu}(\sigma) d \sigma\right\rangle=\vec{x}_{0}^{\mu}+\frac{\vec{p}_{0}^{\mu}}{m} s+\frac{1}{2} c g^{\mu} s^{2} . \\
\sigma_{x}^{2}=\left\langle x_{\mu} x^{\mu}\right\rangle-\vec{x}_{\mu} \vec{x}^{\mu}=\left\langle\left(\zeta^{\mu}+\frac{1}{m} \int_{s_{0}}^{s}\left(\eta^{\mu}+m c g^{\mu} s+m c g^{\mu} \int_{\sigma_{0}}^{\sigma} \xi\left(\sigma^{\prime}\right) d \sigma^{\prime}\right) d \sigma\right)^{2}\right\rangle-\left(\vec{x}_{0}^{\mu}+\frac{\vec{p}_{o}^{\mu}}{m} s+\frac{1}{2} c g^{\mu} s^{2}\right)^{2}= \\
=\sigma_{x 0}^{2}+\sigma_{x 0}^{2}\left(\frac{s}{m}\right)^{2}+c^{2} g_{\mu} g^{\mu} \int_{s_{0}}^{s} \int_{s_{0}}^{s}\left(\int_{\sigma_{0}}^{\sigma} \int_{\sigma_{0}^{\prime}}^{\sigma^{\prime}} \xi(\chi) \xi\left(\chi^{\prime}\right) d \chi d \chi^{\prime}\right) d \sigma d \sigma^{\prime}=\sigma_{x 0}^{2}+\sigma_{x 0}^{2}\left(\frac{s}{m}\right)^{2}+\frac{1}{3} \kappa c^{2} g_{\mu} g^{\mu} s^{3} .
\end{gathered}
$$

From (120), (121) one can also obtain the distribution function (112) in the subspace of 4-coordinates.

The expressions (117), (121) also allow one to get the probability density (108), in which

$$
\begin{gathered}
\sigma_{x}^{2}=\sigma_{x 0}^{2}+\frac{\sigma_{x 0}^{2}}{m^{2}} s^{2}+\frac{1}{3} \kappa c^{2} g_{\mu} g^{\mu} s^{3}, \\
\sigma_{p}^{2}=\sigma_{p 0}^{2}+\kappa m^{2} c^{2} g_{\mu} g^{\mu} s, \\
\sigma_{x p}=\frac{\sigma_{p 0}^{2}}{2 m}+\frac{1}{2} \kappa c^{2} g_{\mu} g^{\mu} s^{2} .
\end{gathered}
$$

For a one-dimensional case with the initial conditions $t_{0}=0, x_{0}=0$, from (122)-(124) it follows

$$
\begin{gathered}
\sigma_{x}^{2}=\sigma_{x 0}^{2}+\frac{\sigma_{x 0}^{2}}{m^{2}}\left(c^{2} t^{2}-x^{2}\right)+\frac{1}{3} \kappa c^{2} g^{2} \sqrt{\left(c^{2} t^{2}-x^{2}\right)^{3}} \\
\sigma_{p}^{2}=\sigma_{p 0}^{2}+\kappa m^{2} c^{2} g \sqrt{\left(c^{2} t^{2}-x^{2}\right)} \\
\sigma_{x p}=\frac{\sigma_{p 0}^{2}}{2 m}+\frac{1}{2} \kappa c^{2} g^{2}\left(c^{2} t^{2}-x^{2}\right)
\end{gathered}
$$

Note that variances of the 4-coordinate and momentum as well as of the correlation function grow as $s$ and $t$ increase. Let us find now the Shannon entropy for the distribution functions (108),(112), (113): 


$$
\begin{gathered}
S_{x}=-\int_{-\infty}^{+\infty} F\left(x^{\mu}\right) \ln F\left(x^{\mu}\right) d x^{\mu}=\frac{1}{2}+\ln \left(\sqrt{2 \pi} \sigma_{x}\right) \\
S_{x}=-\int_{-\infty}^{+\infty} F\left(x^{\mu}\right) \ln F\left(x^{\mu}\right) d x^{\mu}=\frac{1}{2}+\ln \left(\sqrt{2 \pi} \sigma_{x}\right), \\
S_{p}=-\int_{-\infty}^{+\infty} F\left(p^{\mu}\right) \ln F\left(p^{\mu}\right) d p^{\mu}=\frac{1}{2}+\ln \left(\sqrt{2 \pi} \sigma_{p}\right) .
\end{gathered}
$$

\section{Conclusion}

The obtained results (90), (94), (99), and (108) demonstrate a drastic difference between the RMM and the Newtonian AMM. Instead of Newton's $1^{\text {st }}$ law of motion (in an inertial reference frame, a free body either is at rest or moves at a constant velocity), the equation (94) expresses the law of the loss of information on the state of an isolated body with the time elapsed after the last measurement. Only the maximum of the distribution function moves uniformly, but the probability at its maximum steadily decreases with time, whereas the variance steadily increases under the law (95). Thus, in actual practice, P. Laplace could not had predicted the future of the Universe. Moreover, he could not had predicted even the collision of two sufficiently distant (at a distance $X^{*}$ ) real billiard balls a certain finite time $(\tau)$ after the measurement (at the time $t_{0}$ ) of positions and velocities of these balls aimed at each other head-on. And at distances $x<x^{*}$, when a grazing collision of the balls is still possible, Laplace could only had calculated, with some probability less than 1 , the angles of scattering. In the context of the RMM, the global entropy grows steadily even for a system of two real particles. Hence, in order to deduce the 2nd law of thermodynamics from the RMM, one should not make a non-constructive operation of a passage to the thermodynamic limit.

As regards the equation (88), it is identical to the Liouville equation for a straight-line motion of the particle and, hence, it is equivalent to both Newton's 1st and 2nd laws for an abstract point-like particle of mass $m$ moving in a tube of a constant cross-section. Thus, the equation (90) agrees with the previously obtained result (77). Here the global entropy does not grow, that means that from the abstract Newtonian dynamics one cannot obtain the 2 nd law of thermodynamics for abstract point-like structure-free particles.

It is interesting, that the Boltzmann kinetic equation (1) has the structure of the equation (84) without diffusion term, but with the collision integral, which plays the role of the "jump integral" in the right part of (84). Thus, paraphrasing G. A. Martynov, we can say that just the Boltzmann equation (1) is the exact equation of RMM, while Newton's mechanics, Poincaré's theorem, Liouville's equation and all of the BBGKY hierarchy are a quite abstract (approximate) model of Universe, although all of them have the right to exist as beautiful, informative, and very important abstract mathematical constructions.
It is characteristic that the result of T. Nishigory [20] has not rouse the scientific community to correct the classical mechanics for non-stable motions. Very similar results were obtained by other authors. For example, E.T. Jaynes [28] has shown that for the validity of the Boltzmann H-theorem the energy of monatomic system should exceed some minimal value. It means that the entropy grows only in the case of non-stable motion. On the contrary, the H-theorem is inconsistent with Newton's mechanics only in the case of stable systems, for which, according to A. Poincaré, the incomplete Liouville equation (15) is valid. Then, J. Keizer [29] has demonstrated that the entropy grows only in systems with a non-singular transition matrix, i.e. the entropy does not grow, if the variance of distribution function is zero. At last, A.K. Rajagopal et al. [30] have obtained a result, very similar to that of T. Nishigory [20], that the Boltzmann $\mathrm{H}$-theorem does not valid in the case of initial distributions like the Dirac delta-function.

\section{REFERENCES}

[1] Silin V. P. Vvedenie v kineticheskyu teoriyu gasov.(Russ.) Moscow, Nauka, 1971.

[2] Synge J. L. Classical Dynamics. Berlin-Gцttingen-Heidelberg: Springer, 1960.

[3] Gurov K. P. Osnovanija kineticheskoy teorii. Metod N.N. Bogolubova.(Russ.) - Moscow: Nauka, 1966.

[4] Bogolubov N. N. Problemy dynamicheskoy teorii v statisticheskoy fisike.(Russ.) - Moscow: Gostechizdat, 1946.

[5] Martynov G. A. // Uspechi fisicheskikh nauk, 1996. V. 166, N.10. P. 1105.

[6] Zaslavskii G. M., Sagdeev R. Z.. Vvedenie v nelineynuy fiziku: ot mayatnika do turbulentnosti i haosa. (Russ.) Moscow: Nauka, 1988.

[7] Gaponov-Grekhov A. V., Rabinovitch M. I. // Priroda, 1981, N.2. P. 54.

[8] Landau L. D., Lifshitc E. M. Statisticheskaja fisika.(Russ.) Moscow: Nauka, 1964, P. 48.

[9] Nemytskii V. V., Stepanov V. V. Kachestvennaja teorija differencial'nych uravnenij. (Russ.) - Moscow-Leningrad, 1947.

[10] Erugin N. P. Kniga dlja chtenija po obstchemu kyrsy differencial'nych uravnenij.(Russ.) - Minsk: "Nauka i technika", 1970.

[11] Arnold V. I. // Priroda, 1987. N.8, P. 5.

[12] Kuznetsov I. V. // Great Soviet Encyclopedy (3rd Ed., Russ.), 1972. V. 8, P. 146.

[13] Katastroph teorija. // Great Soviet Encyclopedy (2nd Ed., Russ.), 1953. V. 20, P. 365.

[14] Ogurtsov A. P. // Great Soviet Encyclopedy (3rd Ed., Russ.), 1972. V. 10, P. 186. 
[15] Vainberg M. M., Trenogin V. A. Teorija vetvlenija reshenij nelineynych uravnenij.(Russ.) - Moscow: Nauka, 1969.

[16] Bellman R. B. Stability theory of differential equations. N.-Y.-Toronto-London, 1953.

[17] Chetaev N.G. Ustoijchivost' dvizhenija. (Russ.) - Moscow: Nauka, 1965.

[18] Demidovitch B. P. Lekcii po matematicheskoy teorii ustoychivosti. (Russ.) - Moscow: Nauka, 1967.

[19] Sinay Ja. G. // Priroda, 1981. N.3, P. 72.

[20] Nishigory T. // Progr. Theor. Phys., 1979. V. 62, N.4. P. 1156.

[21] Gnedenko B. V. Kurs teorii verojatnostej. (Russ.) - Moscow: Fizmatgiz, 1961.

[22] Sveshnikov A. A. Prikladnye metody teorii slutchainych funkcii. (Russ.) - Moscow: Nauka, 1968.

[23] Gikhman I. I., Skorokhod A. V. Stochasticheskie differencial'nye uravnenija. (Russ.) - Kiev: Naukova Dumka, 1968.

[24] Skorobogatov G. A. // Vestnik St.Petersburg's Universiry (Ser. 4), 2007. No.4. P.3.
[25] Tertytchny-Dauri V. Yu. Stokhastitcheskaja mekhanika. (Russ.) - Moscow, 2001.

[26] Matveev N. M. Metody integrirovanija obyknovennych differencial'nych uravnenii.(Russ.) - Leningrad, 1955.

[27] Almeida C., Jabs A. // Am. J. Phys., 1984. V.52, N.10. P. 921.

[28] Janes E. T. //Phys. Rev. (A), 1971. V. 4, N.2. P. 747.

[29] Keizer J. // J. Statistical Phys., 1972. V. 6, N. 2/3/ P. 67.

[30] Rajagopal A. K., Teitler S., Ngai K. L., Rendell R. W. // Phys. Rev.(A), 1984. V. 29, N. 2. P. 969.

[31] Jordan T. F. Spreading velocity of relativistic wave packet // Amer. J. Phys., 1986. V. 54, N.2. P. 182.

[32] Blinder S. M. Evolution of a Gaussian wavepacket // Amer. J. Phys., 1968. V. 36, N.6. P. 525-527.

[33] Bondar D. I., Lompay R. R., Liu W.-K. Quantum mechanics of a free particle from properties of the Dirac delta function // Amer. J. Phys., 2011. V. 79, N.4. P. 392-394.

[34] Vandegrift G. Accelerating wave packet solution to Schrödinger's equation // Amer. J. Phys., 2000. V. 68, N.6. P. 576-577. 